\title{
HEART: Unrelated Parallel Machines Problem with Precedence Constraints for Task Scheduling in Cloud Computing using Heuristic and Meta-Heuristic Algorithms
}

\author{
Amit Kumar Bhardwaj ${ }^{1,2}$, Yuvraj Gajpal ${ }^{2}$, Chirag Surti ${ }^{3}$, Sukhpal Singh Gill ${ }^{4}$ \\ ${ }^{1}$ L.M. Thapar School of Management, Thapar Institute of Engineering \& Technology, Dera Bassi Campus, Punjab, India \\ ${ }^{2}$ Supply Chain Management, Asper School of Business, University of Manitoba, Canada \\ ${ }^{3}$ Dept. of Information System, Analytics and Supply Chain Management, Rider University, USA \\ ${ }^{4}$ School of Electronic Engineering and Computer Science, Queen Mary University of London, London, UK \\ akbhardwaj@thapar.edu,yuvraj.gajpal@umanitoba.ca, cssurti@gmail.com, s.s.gill@qmul.ac.uk
}

\begin{abstract}
Cloud computing is becoming a profitable technology because of it offers cost-effective IT solutions globally. A well-designed task scheduling algorithm ensures the optimal utilization of clouds resources and reducing execution time dynamically. This research paper deals with the task scheduling of inter-dependent subtasks on unrelated parallel computing machines in a cloud computing environment. This paper considers two variants of the problem-based on two different objective function values. The first variant considers the minimization of the total completion time objective function while the second variant considers the minimization of the makespan objective function. Heuristic and meta-heuristic (HEART) based algorithms are proposed to solve the task scheduling problems. These algorithms utilize the property of list scheduling algorithm of unrelated parallel machine scheduling problem. A Mixed Integer Linear Programming (MILP) formulation has been provided for the two variants of the problem. The optimal solution is obtained by solving MILP formulation using A Mathematical Programming Language (AMPL) software. Extensive numerical experiments have been performed to evaluate the performance of proposed algorithms. The solutions obtained by the proposed algorithms are found to out-perform the existing algorithms. The proposed algorithms can be used by cloud computing service providers (CCSPs) for enhancing their resources utilization to reduce their operating cost.
\end{abstract}

Keywords: Scheduling; Heuristics; Resource Optimization; Cloud Computing; Metaheuristic.

\section{Introduction}

Cloud computing is gaining popularity due to its ability for delivering cost-effective cloud services which can bring a win-win situation for the end users as well as for the service providers (Khiat et al. (2020)). In 
the cloud computing environment, the remote users can obtain computational resources over the Internet in scalable fashion based on their requirement (Gabriel and Moallemi (2020)). Cloud computing has become a backbone for the IT infrastructure of different industries and organizations viz. education, weather forecasting, hedge funding, e-commerce and big data solution (Gill et al. 2019, Kaur et al. 2019). Kochan et al. (2018) used a cloud computing based framework in the domain of hospital supply chain to enhance the performance of demand and supply of healthcare items (Gill et al. 2019, Buyya et al. 2018, Islam et al. 2020).

According to the enterprise cloud computing survey, the top four cloud service providers are Microsoft Azure with $23 \%$ share, Amazon Web Services (AWS) with 22\% share, Google Cloud with 21\% share, and IBM Cloud with 17\% share (Enterprise Cloud Computing Survey (2016)). This survey forecast that about $90 \%$ of enterprises will increase their annual spending on cloud computing. Also, SaaS-based applications are expected to grow by $18 \%$, and Infrastructure/ Platform as a service is expected to grow by $27 \%$ annually. The survey further indicates the increases in the efficiency of the enterprises through the use of cloud computing solutions. According to the Forbes magazine report, the total turnover of the cloud computing industry was $\$ 67$ Billion in 2015 , which is expected to grow by $\$ 162$ billion in 2020 (The Changing Faces of the Cloud (2017)). The report further reveals that Cloud Computing will impact the business considerably in near future. The Cloud Computing Services Providers (CCSPs) are competing with each other to capture the market share (Bowen et al. (2015)). Hence, the success of a CCSP depends upon the cost-effective offerings to their clients (Casini et al. (2020)).

In a cloud computing environment, a cloud is considered as a cluster of many distributed computers (Germain \& Rana (2009), Tuli et al. (2020)). A physical computer can have more than one Virtual Machines (VMs) residing on it for a parallel execution of different tasks (Gill et al. (2020)). Thus, the cloud computing system can be considered as a system of parallel VMs (Saif et al. (2020)). An end user can use the cloud resources in the form of a lease from the cloud service providers (Srirama and Ostovar (2018)). The VMs can be leased at the price of 10 cents per hour (Li et al. (2012)). In a cloud computing 
environment, millions of users submit their billions of tasks for processing on unrelated virtual parallel machines environment (Deming and Liu (2020)). In unrelated parallel machines environment, the execution time of a task is considered to be different for different machines (Vallada et al. (2019)). An unequal execution time of tasks over different machines arises because each machine has different processing speed,

memory or complexity (Ezugwu (2019)). This paper deals with the design of efficient algorithms to achieve the cost-effective solution for cloud computing task scheduling problem using precedence constraints.

\subsection{Motivation and Our Contributions}

The main motivation behind this research work is to consider a scheduling problem where unrelated VMs are responsible for processing independent tasks. A task consists of a series of subtasks (Tsai et al. (2013)). A successor subtask can be started only after the completion of its predecessor subtask. The subtasks can be executed independently in the same machine or in a different machine. The execution time of each machine is known in advance. Each machine has known release time (i.e., the available time for execution of existing tasks) due to the ongoing process of other jobs. In this research paper, we have considered two problem variants for two different objective functions. The first variant of the problem considers the minimization of the total completion time objective while the second variant of the problem considers the minimization of the makespan objective. A Mixed Integer Linear Programming (MILP) formulation has been provided for the proposed variants of the problem. The MILP formulation is solved to obtain an optimal solution for small problem instances. We proposed HEuristics and metA-heuRisTic (HEART) algorithms to solve the bigger problem instances. The proposed algorithms are compared with the similar algorithms available in the literature. The benchmark problem instances are introduced to perform the numerical experiments. These problem instances can be used in future research to compare the performance of different algorithms.

\subsection{Article Organization}

The rest of the paper is organized as follows. Section 2 provides a literature review of related research work on task scheduling in cloud computing domain along with the literature review of parallel machines 
scheduling problems. Section 3 provides the problem description and formulation of the proposed problem, whereas Section 4 describes the proposed algorithms. In Section 5, the results of the proposed algorithms are compared with the existing algorithms. Finally, Section 6 provides the conclusion of this research paper.

\section{Related Work}

The related work section is divided into two subsections: 1) task scheduling and 2) unrelated parallel machines.

\subsection{Task Scheduling in Cloud Computing}

In the last decade, application of scheduling algorithms in the cloud computing environment took the momentum with the aim of improving the resource utilization (Bhatt et al. (2020)). Gill \& Buyya (2018) discussed the resource provisioning for workloads in the parallel computing environment of clouds. Fang et al. (2010) discussed the load balancing problem in cloud computing environment to meet the Quality of Service (QoS) requirements. Many researchers considered the genetic algorithm to solve QoS-oriented scheduling problems in cloud computing (Dutta \& Joshi (2011), Jang et al. (2012), Liu et al. (2013), and Li \& Peng (2011)). Pandey et al. (2010) used Particle Swarm Optimization (PSO) for job scheduling to minimize the computation and data transmission costs. Tsai et al. (2013) considered parallel cloud computing services with different processing capacity to perform subtasks. They included processing and receiving cost in their model with the aim to minimize the cost and makespan simultaneously. These research papers indicate the popularity of scheduling issues in cloud computing environment due to its ability to improve resource utilization. Also, there are many algorithms available in the literature to solve the scheduling problem in the production environment. However, the above-mentioned paper did not utilize the existing algorithms/properties form a production scheduling problem to solve the cloud computing scheduling problem. One of the aims of this paper is to relate the existing scheduling model from the production environment to the scheduling model in the cloud computing environment.

The problem considered in this paper resembles with the parallel machine scheduling problem. The parallel machine scheduling problem has been proved to be NP-hard (Cook (1971) and Garey Johnson (1979)). The parallel machine problem can be classified under three categories on the basis of machine configuration: 
uniform machine configuration, heterogeneous machine configuration and unrelated machine configuration. In uniform machine configuration, the processing time of a task is the same for all machines. In heterogeneous machine configuration, the processing time of a task depends on machine speed. In unrelated machine configuration, the processing time of a task is different for different machines. The problem considered in this paper resembles with unrelated parallel machine problems, therefore, the remainder of the literature review discusses only unrelated parallel machine scheduling problems.

\subsection{Unrelated Parallel Machine Scheduling}

Luis \& Ruiz (2010) and Lin et al. (2011) proposed metaheuristics to solve the unrelated parallel machine problems to minimize the makespan objective. Luis \& Ruiz (2010) proposed iterative greedy local search based metaheuristic to solve the problem. Lin et al. (2011) proposed an artificial immune system, which combines the feature of the artificial immune system and simulated annealing. Lin et al. (2013) extended their work for the multi-objective problem by proposing a Genetic Algorithm (GA) to find the non-dominated solutions to minimize the makespan, the total weighted completion time, and the total weighted tardiness objectives. Other research papers in literature (Ezugwu et al. (2019), Vallada et al. (2019), Lei and Liu (2020)) considered variants of unrelated parallel machine problems such as setup time, batch processing, additional resources etc.

Jose et al. (2017) proposed Job Scheduling Technique (JST) considered non-identical job sizes and unequal ready times over the unrelated parallel batch processing machines to minimize makespan objective. They developed many scheduling heuristics based on first-fit, best-fit and earliest job ready time rules to solve the problem. Shahvari \& Logendran (2017) proposed an enhanced Tabu Search Algorithm (TSA) to solve the problem and considered batch processing in unrelated parallel machines to minimize a linear combination of total weighted completion time and the total weighted tardiness objectives. Joo \& Kim (2015) considered setup time and production-availability in unrelated parallel machines to minimize the total completion time objective. They proposed a hybrid genetic algorithm to solve the problem. Cheng \& Huang (2017) addressed an unrelated parallel machine scheduling problem for jobs with distinct due dates and dedicated machines to minimize the total earliness and tardiness objectives. They developed a modified GA with a distributed 
release time control mechanism to solve the problem. Rodney et al. (2015) considered unrelated parallel machines with sequence dependent setup times to minimize the makespan. They developed a Variable Neighborhood Descent (VND) metaheuristic to solve the problem. Luis et al. (2017) considered unrelated parallel machine problem with additional resources to minimize the makespan objective. They proposed a mathematical model based on linear programming formulation to solve the problem. Further, they combined metaheuristic strategies with a linear programming model to solve the bigger problem instances. Oleh \& Lars (2016) considered scheduling problems in flexible job shops in an unrelated parallel machines environment to minimize the total weighted tardiness objective. They proposed an iterative local search to solve the problem.

Wang et al. (2020) developed an Optimal Charging Scheduling (OCS) technique for electric vehicles considering the impact of renewable energy sources, which uses MILP to optimize execution time. Deng et al. (2020) proposed a MILP based Two-Stage Load (TSL) scheduling approach for building load's peak-toaverage ratio reduction and improves execution time. These prior works such as Wang et al. (2020) and Deng et al. (2020) use MILP with a limited perspective. None of them considered precedence constraints in their problem. The unrelated parallel machine problem, with precedence constraints, is considered by Herrmann et al. (1997), Liu \& Yang (2011), Afzalirad \& Rezaeian (2016) and Gacias et al. (2010). The problem presented in this paper considers that many independent tasks consist of numerous inter-dependent subtasks. Each subtask can be processed independently but it can be started only after processing of predecessor subtask. Thus, the problem discussed in this paper can be considered as a special case of unrelated parallel machine scheduling problem with precedence constraints. Gacias et al. (2010) considered scheduling problem with precedence constraints and sequence-dependent setup times to minimize the total completion time and maximum lateness objectives independently. They proposed a branch-and-bound based exact algorithm and limited discrepancy based heuristic method to solve the problem. Afzalirad \& Rezaeian (2016) considered resource constrained unrelated parallel machine scheduling problem with sequencedependent setup time, precedence constraints and machine eligibility restrictions to minimize the makespan objective. They developed a GA and artificial immune system (AIS) to solve the problem. 
Herrmann et al. (1997) considered unrelated parallel machine scheduling problem with precedence constraints to minimize makespan objective. Herrmann et al. (1997) highlighted the problem as an application of office scheduling problem where workers perform different interdependent tasks with different skill sets for each subtask. They proposed a look-ahead based HH heuristic to solve the problem. The HH heuristic is based on scheduling a task in each iteration, which could lead to a late schedule of some tasks in the future. Liu and Yang (2011) proposed a serial schedule (SS) heuristic to solve unrelated machine problem with precedence constraints for minimizing the makespan objective. The SS heuristic assigns a task to the earliest available machine iteratively. They compared SS algorithm with HH algorithm of Herrmann et al. (1997). Their numerical experiment showed the better performance of SS heuristic as compared to the HH heuristic.

The algorithm proposed by Gacias et al. (2010) and Afzalirad \& Rezaeian (2016) cannot be used to solve the problem considered in this paper because they considered additional constraints. However, the algorithm proposed by Herrmann et al. (1997) and, Liu \& Yang (2011) can be used to solve the problem considered in this paper. We propose a simple heuristic and ant colony based metaheuristic to solve the problem. In the numerical experiment, we compare the performance of proposed algorithms with the SS heuristic of Liu \& Yang (2011) and HH heuristic of Herrmann et al. (1997).

\subsection{Critical Analysis}

As discussed above, none of the above-mentioned papers considered precedence constraints in their unrelated parallel machines problem. Existing works are considering tasks independently, which leads to poor scheduling decisions, as shown in the evaluation section. Wang et al. (2020) and Deng et al. (2020) use MILP without considering. Hence, the precedence constraints-based MILP and heuristic/meta-heuristic approaches out-perform the baseline models. The current work shows a proof-of-concept of the novel approach and establishes that the proposed approach out-performs in a fundamental computing platform. This research work performs task scheduling of inter-dependent subtasks on unrelated parallel computing machines in a cloud computing environment using heuristic and meta-heuristic algorithms. We have performed the evaluation of our research work by considering all the possible performance parameters. Table 1 shows the 
comparison of proposed work (HEART) with existing techniques based on important parameters.

Table 1: Comparison of our proposed work (HEART) with related works

\begin{tabular}{|c|c|c|c|c|c|c|c|c|c|c|c|c|c|}
\hline \multirow[t]{2}{*}{ Work } & \multirow{2}{*}{$\begin{array}{c}\text { Heuristic } \\
\text { Optimization }\end{array}$} & \multirow{2}{*}{$\begin{array}{c}\text { Multi- } \\
\text { Scale } \\
\text { System }\end{array}$} & \multirow[t]{2}{*}{ Dataset } & \multirow{2}{*}{$\begin{array}{c}\text { Unrelated } \\
\text { Parallel } \\
\text { Machines }\end{array}$} & \multirow{2}{*}{$\begin{array}{l}\text { Precedence } \\
\text { Task } \\
\text { Constraints }\end{array}$} & \multirow{2}{*}{$\begin{array}{c}\text { Heuristic and } \\
\text { Meta-Heuristic } \\
\text { Algorithms }\end{array}$} & \multirow{2}{*}{$\begin{array}{c}\text { Cloud } \\
\text { Computing }\end{array}$} & \multicolumn{6}{|c|}{ Evaluation Parameters } \\
\hline & & & & & & & & MKS & TCT & RPD & CPU & PG & ST \\
\hline $\begin{array}{l}\text { HH Heuristic (Herrmann et } \\
\text { al. (1997)) }\end{array}$ & $\sqrt{ }$ & $\sqrt{ }$ & Small & & $\sqrt{ }$ & & & $\sqrt{ }$ & & & & & \\
\hline $\begin{array}{c}\text { SS Heuristic (Liu \& Yang } \\
\text { (2011)) }\end{array}$ & $\mathrm{V}$ & & Small & & $\sqrt{ }$ & & $\sqrt{ }$ & $\sqrt{ }$ & & & & & \\
\hline JST (Jose et al. (2017)) & $\sqrt{ }$ & & Small & $\sqrt{ }$ & & & & $\sqrt{ }$ & & & & & \\
\hline $\begin{array}{c}\text { TSA (Shahvari \& } \\
\text { Logendran (2017)) }\end{array}$ & & & Small & $\sqrt{ }$ & & & & $\sqrt{ }$ & & & & & \\
\hline OCL (Wang et al. (2020)) & & $\sqrt{ }$ & Small & & & & & $\sqrt{ }$ & & & & & \\
\hline TSL (Deng et al. (2020)) & & $\sqrt{ }$ & Small & & & & & $\sqrt{ }$ & & & & & \\
\hline HEART (this work) & $\sqrt{ }$ & $\sqrt{ }$ & Small and Large & $\sqrt{ }$ & $\sqrt{ }$ & $\sqrt{ }$ & $\sqrt{ }$ & $\sqrt{ }$ & $\sqrt{ }$ & $\sqrt{ }$ & $\sqrt{ }$ & $\sqrt{ }$ & $\sqrt{ }$ \\
\hline
\end{tabular}

Abbreviations: MKS: Makespan value produced by an algorithm, TCT: Total completion time value produced by an algorithm, RPD: Relative percentage deviation of an algorithm from the best solution, CPU: CPU time consumed by an algorithm, PG: Percentage gap of an algorithm form the optimal solution and ST: Scheduling Time

\section{System Model and Problem Formulation}

The section discusses the cloud model and problem formulation.

\subsection{Cloud Model}

The proposed algorithms in this paper can be deployed in real cloud platforms to allow efficient task scheduling in unrelated machines. This is quite evident in modern systems due to variation in compute performance, bandwidth availability and dynamic resource consumption statistics. Thus, we describe a large-scale distributed cloud platform model suitable for the proposed heuristic and meta-heuristic algorithms. The system model is shown in Figure 1. The design strategy as illustrated in the figure is as follows: The data is acquired from the Data Acquisition layer which consists of API gateways, IoT devices including sensors and Point of Scale (PoS) systems (AVAC, Tuli et al. 2020). This data is encapsulated in a task using gateways to be sent to Computing and Communication layer. Herein, the cloud nodes communicate using light-weight message passing approaches like MQTT to share task data and computational meta-data. MQTT is a machine-to-machine (M2M)/'Internet of Things" connectivity protocol (Hunkeler et al. 2008). The Analytics layer resides in one of the cloud nodes which handles the complete system and is responsible for monitoring and scheduling of tasks and cloud machines. The final task results are sent to the end user from the Analytics layer using alerts, web-portals or gateway applications (Mancini et al. 2019). 


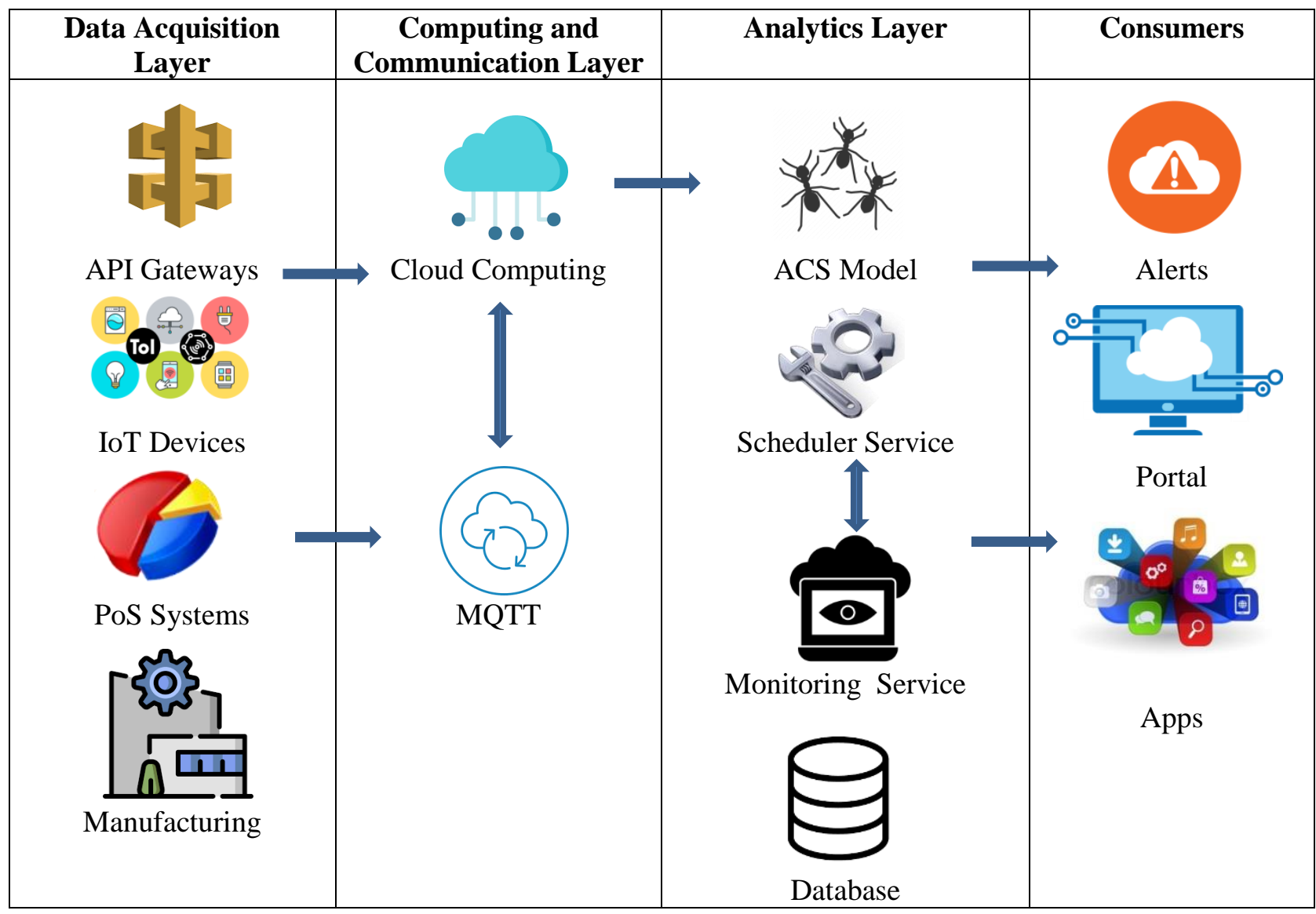

Figure 1: System Model

\subsection{Problem Formulation}

The problem considered in this paper deals with a situation in which a cloud computing scheduler receives $n$ independent tasks for executing at $m$ resources. Each task consists of a series of subtasks and is acceptable for processing on any resources. The cloud computing scheduler wants to assign $n t$ inter-dependent subtasks from set $T=\left(T_{1}, T_{2}, T_{3}, \ldots T_{i}, \ldots T_{n t}\right)$, to $m$ available resources $R=\left(R_{1}, R_{2}, \ldots, R_{m}\right)$. A task $i$ consists of $n^{i}$ inter-dependent series of subtasks denoted by set $T^{i}=\left(T_{l}, \ldots T_{l+n^{i}}\right)$, where $T_{l}$ and $T_{l+n^{l}}$ are the first and last subtask for the task $l$. There is a temporal relationship among subtasks of task $i$, viz. all subtasks are performed in a sequential series. The subtask $T_{l+1}$ can be started only after completion of the sub-task $T_{l}$; the subtask $T_{l+2}$ can be started only after completion of the subtask $T_{l+1}$ and so on. Each subtask has just one predecessor except the first subtask of a task. Let $\operatorname{pred}\left(T_{i}\right)$ denote the predecessor of task $T_{i}$, which can be defined as follows. 


$$
\operatorname{pred}\left(T_{i}\right)=\left\{\begin{array}{cc}
T_{i-1} & \text { if subtasks } T_{j-1} \text { and } T_{j} \text { belongs tothe same job } \\
T_{0} & \text { otherwise }
\end{array}\right.
$$

Here $T_{0}$ is an imaginary subtask with zero process time. We assume that this subtask has already been completed. A subtask $T_{i}$ can be executed to any cloud resources $R_{k} \subseteq R$, however, it can be started only after the completion of predecessor subtask $\operatorname{pred}\left(T_{i}\right)$.

The process time $P_{i k}$ for executing the subtask $T_{i}$ on resource $R_{k}$ is known in advance. A resource $R_{k}$ is available for processing any subtask only after time $t_{k}$ because the resources are assumed to be, at the time, executing previously assigned subtask. A subtask-preemption is not allowed and resources are not allowed to process more than one subtask at a time. A subtask is executed on a single resource at a time and the given resources are available continuously. The problem involves assigning subtasks to appropriate resources in such a way that the specified objective function is minimized. A MILP formulation is provided for the two variants of the problem. The first variant of the problem considers the minimization of the total completion time objective function and the second variant of the problem considers the minimization of the Makespan objective. The first problem is denoted as Problem 1 and the second problem is denoted as Problem 2. Two variants of the problem are formulated using the following decision variables.

\section{Decision variables:}

$F_{j k} \quad$ Completion time on resource $j$ for a subtask scheduled at position $k$,

$X_{i j k} \quad$ Binary variable taking value 1 if subtask $i$ is assigned to resource $j$ at position $k ; 0$ otherwise,

$C_{i} \quad$ Completion time of task $i$,

$C_{\max }$ Makespan of the optimal solution.

\section{Problem 1: Minimization of total completion time}

$$
\operatorname{Min} \quad Z=\sum_{i=1}^{n} C_{i}
$$




$$
\begin{aligned}
& \sum_{j=1}^{m} \sum_{k=1}^{n} X_{i j k}=1 \quad \forall i \in T \\
& \sum_{i=1}^{n} X_{i j k} \leq 1 \quad \forall j \in R, k \in L \\
& F_{j 1}>=t_{j}+\sum_{i=1}^{n} X_{i j 1} \times P_{i j} \forall j \in R ; \\
& F_{j k} \geq F_{j k-1}+\sum_{i=1}^{n} X_{i j 1} \times P_{i j} \forall j \in R, k \geq 2 \\
& C_{i} \geq F_{j k}-M\left(1-X_{i j k}\right) \quad \forall i \in T, \forall j \in R, k \in L \\
& C_{i} \geq C_{p r e d\left(T_{i}\right)}+\sum_{j=1}^{m} \sum_{k=1}^{n} p_{i j} X_{i j k} \quad \forall i \in T \\
& C_{0}=0 \\
& X_{i j k} \in\{0,1\}, \forall i \in T, j \in R, k \in L
\end{aligned}
$$

Equation (1) provides the expression for the minimization of the total completion time. Equation (2) ensures that a task $T_{i}$ is scheduled on one resource and one position only. Equation (3) ensures that a maximum one subtask can be assigned for a given resource at a given position. Equation (3) also implies the possibility of not assigning any subtask in a given resource at a given position. Equation (4) calculates the completion time for the task scheduled at first position on resource $j$, while the equation (5) calculates the completion time for other positions. Equation (6) provides the completion time calculation for task $i$. Equations (7) and (8) preserve the predecessor constraints among different subtasks. Finally, equation (9) enforces binary condition for decision variable $X_{i j k}$.

\section{Problem 2: Minimization of Makespan}

$$
\text { Min } \quad Z=C_{\max }
$$

\section{Subject to}




$$
\begin{aligned}
& \sum_{j=1}^{m} \sum_{k=1}^{n} X_{i j k}=1 \quad \forall i \in T \\
& \sum_{i=1}^{n} X_{i j k} \leq 1 \quad \forall j \in R, k \in L \\
& F_{j 1} \geq t_{j}+\sum_{i=1}^{n} X_{i j 1} \times P_{i j} \forall j \in R \\
& F_{j k} \geq F_{j k-1}+\sum_{i=1}^{n} X_{i j 1} \times P_{i j} \forall j \in R, k \geq 2 \\
& C_{i} \geq F_{j k}-M\left(1-X_{i j k}\right) \quad \forall i \in T, \forall j \in R, k \in L \\
& C_{i} \geq C_{p r e d\left(T_{i}\right)}+P_{i j} \quad \forall i \in T \\
& C_{0}=0 \\
& C_{\max } \geq C_{i} \quad \forall i \in T \\
& X_{i j k} \in\{0,1\}, \forall i \in T, j \in R, k \in L
\end{aligned}
$$

Equation (10) provides expression for the minimization of Makespan objective. Equations (11) to (17) are same as equations (2) to (8). The additional constraints (18) provide the calculation of Makespan. Finally, equation (19) enforces the binary constraints for decision variable $X_{i j k}$.

\section{Proposed Algorithms}

In this paper an optimal solution is generated for the total completion time and the Makespan objective using the MILP formulation provided in the previous section. 'A Mathematical Programming Language (AMPL)' software is used to solve the MILP formulation. The MILP formulation can only be solved for small problem instances because the CPU time increases exponentially with increase in problem size. Furthermore, a heuristic and a meta-heuristic are proposed to solve the cloud resource allocation problem for the large problem instances.

The proposed algorithms utilize the list-scheduling dominant property of the unrelated scheduling problem with precedence constraints. A list-scheduling algorithm is an assignment rule that finds a feasible schedule 
for a given order of tasks. The assignment rule considers the tasks one by one from the given list of tasks for assigning them to the machine on the basis of the partial schedule given by the previous scheduled tasks. In the list-scheduling algorithm, decision of scheduled tasks is not changed in future. If a list schedule algorithm evaluates all the feasible schedules related to all possible order of tasks and one of these feasible schedules is guaranteed to find an optimal solution for the problem, then it is said that the list algorithm produces dominant set of solution (Hurnick \& Knust (2001)). In literature, it is said that a list scheduling algorithm finds an optimal solution if it produces a dominant set of solutions. The parallel machine scheduling literature has mainly two list scheduling algorithm: 1) list scheduling algorithm with earliest available machineassignment-rule and 2) list scheduling algorithm with earliest completion time of task-assignment-rule. In the earliest available machine assignment rule based list-scheduling algorithm, next task from the given order of tasks is scheduled on a first available machine. In the earliest completion-time of task-assignment-rule based list-scheduling-algorithm, next task from the given order of tasks is scheduled on a machine where the task completes earliest.

It has been proven that both list scheduling algorithms find an optimal solution for uniform parallel machine scheduling problem with precedence constraints and makespan objective $P \| C_{\max }$ (Hurink \& Knust (2001), Gacias et al. (2010)). However, literature is silent about the list-scheduling algorithm for the total completion-time objective. To the best of our knowledge, optimal list scheduling algorithm for total completion time objective is not available in literature. It can be easily seen that both list scheduling algorithms will produce the same feasible schedule for a given order of tasks in uniform parallel machine problem. However, they will produce different feasible solutions for the unrelated parallel machine scheduling problem with precedence constraints.

Consider an instance with 3 tasks and 2 machines with precedence constraint $J_{1}<J_{3}$ (i.e., task $J_{1}$ precedes task $J_{3}$ ). The process time of tasks on two machines are given in Table 2.

Table 2: Process time of jobs on two machines

\begin{tabular}{|c|c|c|}
\hline Task /Machine & $\mathbf{M}_{1}$ & $\mathbf{M}_{2}$ \\
\hline $\mathbf{J}_{1}$ & 10 & 4 \\
\hline $\mathbf{J}_{2}$ & 6 & 10 \\
\hline $\mathbf{J}_{3}$ & 2 & 10 \\
\hline
\end{tabular}

Preprint Accepted in Journal of Software Practice and Experience Aug 2, 2020 
The optimal solution for this instance is obtained by scheduling task $J_{1}$ in $M_{2}$ and tasks $J_{2}$ and $J_{3}$ in $M_{2}$. The Gantt chart representing optimal solution with Makespan 8 is shown in figure 2.

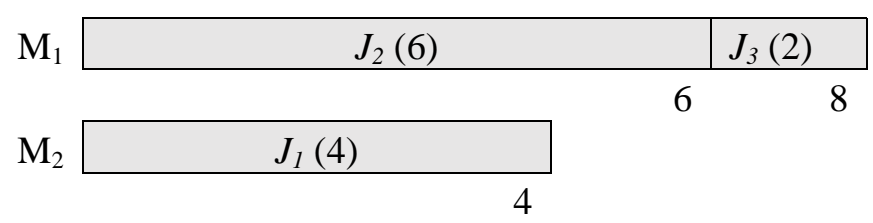

Figure 2: Gantt Chart of Optimal Solution

There can be only three possible lists for the problem satisfying precedence constraints; $\left\{J_{1}-J_{2}-J_{3}\right\},\left\{J_{1}-J_{3}-J_{2}\right\}$ and $\left\{J_{2}-J_{1}-J_{3}\right\}$. The schedule obtained by list scheduling algorithm with earliest available machine assignment rule is given in Figure 3.

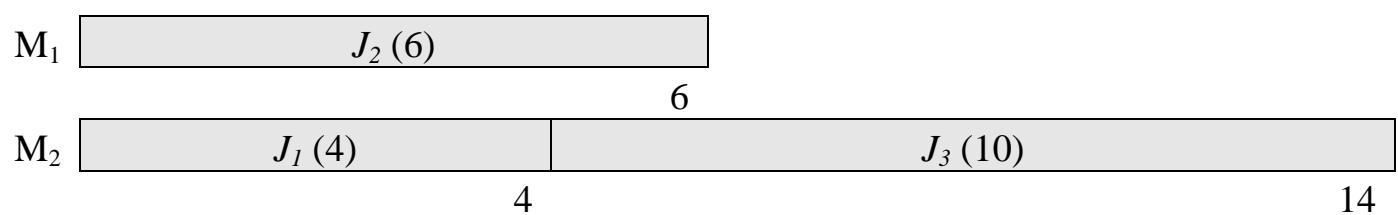

a) Schedule for list $\left\{J_{1}-J_{2}-J_{3}\right\}$

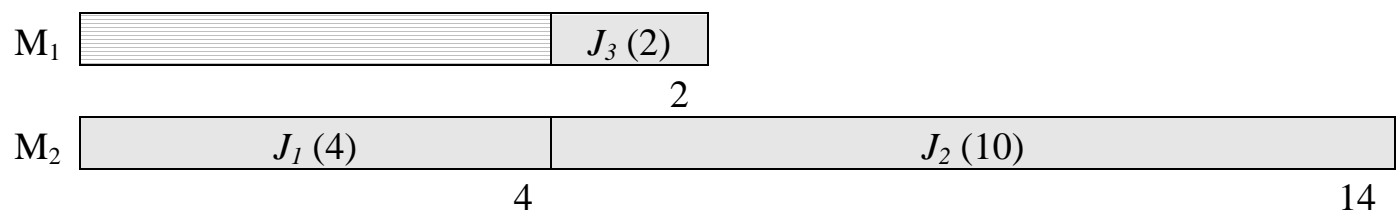

b) Schedule for list $\left\{J_{1}-J_{3}-J_{2}\right\}$

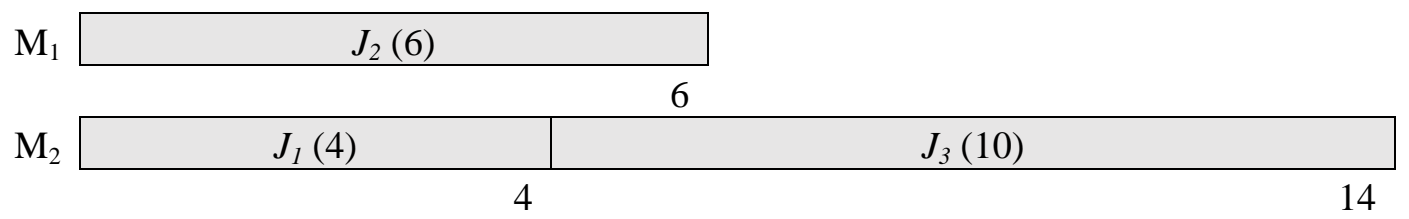

c) Schedule for list $\left\{J_{2}-J_{1}-J_{3}\right\}$

Figure 3: Schedules for list scheduling algorithm with earliest available machine assignment rule

It is clear from Figure 3 that list scheduling algorithm with earliest available machine assignment rule does not produce a dominant set of solution. The schedule obtained by list scheduling algorithm with the earliest completion time of task assignment rule is shown in Figure 4. It appears that the list scheduling algorithm with the earliest completion time of task assignment rule produces a dominant solution. However, we could 
not provide a formal proof of this property. The formal proof is an open-ended research question for future research. We use this property in our proposed algorithms to solve the problem.

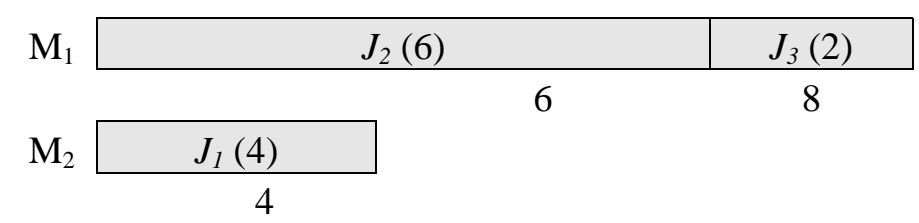

a) Schedule for list $\left\{J_{1}-J_{2}-J_{3}\right\}$

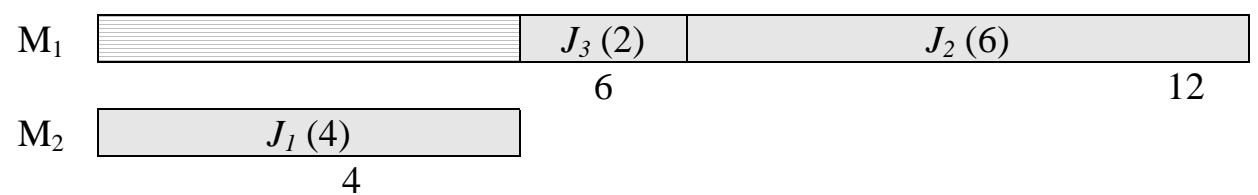

b) Schedule for list $\left\{J_{1}-J_{3}-J_{2}\right\}$

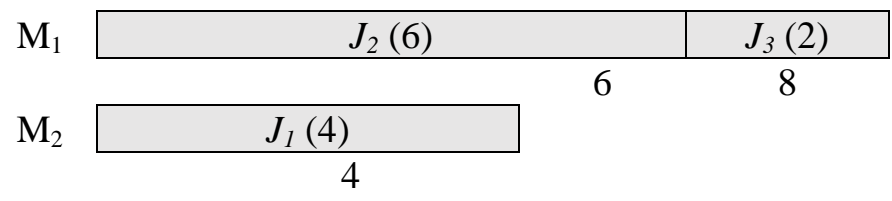

c) Schedule for list $\left\{J_{2}-J_{1}-J_{3}\right\}$

Figure 4 : Schedule for list scheduling algorithm with earliest completion time of task assignment rule

This problem instances can also be used for completion time objective. It can be easily shown that the earliest available machine assignment rule is non-dominant for total completion time objective as well.

\subsection{Earliest Completion Time (EST) based Heuristic}

A heuristic solution method is developed to solve the unrelated parallel machine scheduling problem with precedence constraint. The heuristic partially utilizes the list-scheduling algorithm based on earliest completion time of tasks assignment rule. The proposed heuristic assigns tasks iteratively until all the subtasks are scheduled. In each iteration, all unassigned feasible subtasks i.e., subtasks with assigned predecessor, are evaluated on all the resources. An unassigned subtask with the lowest completion time is selected for the assignment. The detailed description of the heuristic algorithm (Algorithm 1) is provided below. 


\section{Algorithm 1: Earliest Completion Time (EST) based Heuristic Algorithm}

Step 0: Initialize the available time of all the resources as their ready time (i.e., $A_{k}=t_{k} ; k=1, \ldots, m$ ) and completion time of all subtasks to be infinite (i.e. $C_{i}=\infty ; i=1, \ldots, n t$ ). Set the completion time of $0^{\text {th }}$ subtask to be zero (i.e., $C_{0}=0$ ). Initialize unscheduled subtasks set $S=\left\{T_{1}, T_{2}, \ldots, T_{n t}\right\}$.

Step 1: Build the feasible set of subtasks $\Omega$ from the unscheduled subtasks set $S$ whose predecessor subtask has already been scheduled.

Step 2: Evaluate the earliest completion time $E_{i}$ for subtask $T_{i}$ from set $\Omega$.

$$
E_{i}=\min _{k \in M}\left\{\max \left(F_{\operatorname{pred}\left(T_{i}\right)}, A_{k}\right)+P_{i k}\right\}
$$

Step 3: Select the task for scheduling that has minimum earliest completion time $E_{i}$. Determine the resource in which this task can be scheduled for minimum completion time. Assume that subtask $T_{i}$ provides earliest completion time at resource $\mathrm{Rk}$.

Step 4: Schedule the subtask $T_{i}$ at resource $R_{k}$ for processing at time $t=\max \left(C T_{\operatorname{Pred}\left(T_{i}\right)}, A_{k}\right)$. Update the available time $A_{k}$ of resource $R_{k}$ and the completion time $C_{i}$ of task $T_{i}$ as follows.

$$
\begin{aligned}
& A_{k}=t+P_{i k} \\
& C_{i}=t+P_{i k}
\end{aligned}
$$

Step 5: Remove task $T_{i}$ from set $S$.

Step 6: Go back to Step 1 if there is unscheduled task, otherwise stop.

\subsection{Ant Colony System (ACS) Algorithm}

This paper uses the Ant Colony System (ACS) algorithm to solve the problems under consideration. The ACS algorithm is used in many parallel machine scheduling problems. Arnaout et al. (2010) proposed an ACS based algorithm to minimize the Makespan in the parallel machine environment. Behnamian et al. (2009) proposed a hybrid meta-heuristic for a Makespan minimization scheduling problem. They considered the ACS and Variable Neighborhood Search (VNS) algorithms to solve the parallel machine problem. Gao et al. (2013) designed a multi-objective ACS algorithm for the VM placement in the cloud computing environment for the purpose of improving server utilization and power efficiency. Hua et al. (2010) proposed an ACS algorithm for optimizing computing resources allocation problem. Gajpal \& Rajendran (2006) used ACS for minimizing the completion-time variance of jobs in flowshops. Zhang et al. (2018) used ACS in electric vehicle routing problem with recharging stations for minimizing energy consumption. Thiruvady et al. (2013) used ACS in mining domain for a shared resource constrained scheduling problem. Ting \& Chen (2013) used a Multiple Ant Colony Optimization (MACO) algorithm to solve the location-routing problem with capacity constraints on depots and routes. ACS is also used by Thepphakorn et al. (2014) for an 
academic time tabling problem. Hong et al. (2018) used an ACS based heuristic an efficent algorithm for a two-stage supply chain problem with fixed costs. The successful application of ant colony algorithm on solving different combinatorial optimization problem motivated us to use ACS for solving unrelatted parallel machine problem with precedence constraints.

In the ACS algorithm, artificial ants are created to find better solutions to a particular problem by using the information from the solutions of previous iterations. At the end of each iteration, the solutions are stored in the trail intensity of each path. Finally, the ant solutions are generated by using current trail intensity. Detailed explanations and descriptions of the application of ACS can be found in Stützle and Hoos (2000). The fundamental procedure of ACS is shown in Algorithm 2:

Algorithm 2: Ant Colony System (ACS) Algorithm
Step 1: Initialize the trail intensities and parameters
- Generate an ant solution for each ant using the trail intensities.
- Improve ant solution using local search.
- Update trail intensities using elitist ants.
Step 3: Return the best solution found so far.
The trail intensities is denoted as $\tau_{i k}$, which determines the intensity of assigning task $T_{i}$ to
resource $R_{k}$. We initialize the trail intensity $\tau_{i k}=0.01, \forall i \in T, k \in R$.

\subsubsection{Generate an Ant Solution}

In classical, ant colony algorithm a task is selected for scheduling on the basis of trail intensity $\tau_{i k}$ for assigning task $T_{i}$ to resource $k$. The earliest completion time-based list scheduling property seems to provide the optimal solution for the problem considered in this paper. Hence, the proposed ACS utilizes this property for building the ant solution along with the trail intensity of ant algorithm. An ant solution is generated in a similar way of heuristic algorithm is described in Section 4.1 All the steps of generating an ant solution in ACS is similar to the heuristic algorithm except in step 3. In step 3 of heuristic algorithm, subtask $T_{i}$ is Preprint Accepted in Journal of Software Practice and Experience

Aug 2, 2020 
chosen from set $\Omega$ for assignment to resource $R_{k}$. The subtask $T_{i}$ is chosen on the basis of earliest completion time rule. In the proposed ACS, a subtask is chosen using the combination of earliest completion time rule and trial intensity rule. The rules are selected randomly with $90 \%$ probability of earliest completion time rule and $10 \%$ probability on trial intensity rule. The earliest completion time rule is described in step 2 and step 3 of the heuristic algorithm. The trail intensity rule of ant algorithm uses the following probability for selecting $\operatorname{task} T_{i}$.

$$
\mathrm{P}_{i k}=\frac{\tau_{i k}}{\sum_{l \in \Omega} \tau_{l k}}
$$

\subsubsection{Local Search}

Once the solution is constructed by the ant, the ant solution is improved by local search. In the proposed local search scheme, a randomly selected subtask is removed from its original position and re-inserted in all other feasible position. If the best insertion position improves the current solution, then the move is accepted for future evaluation. All the subtasks are evaluated for possible improvement through insertion. The process is repeated if at least one subtask is relocated with improved solution. The process is stopped when none of the subtask insertion is able to improve the solution. The local search uses a speed up mechanism to reduce CPU time by avoiding the evaluation of infeasible insertions. This paper uses two simple properties to identify infeasible insertion places. The first property states that the insertion of a subtask is not feasible anywhere before its immediate predecessor's subtask. The second property states that the insertion of a subtask is not feasible anywhere after its successor's subtask (not only the immediate successor task but all the successor's subtasks).

\subsubsection{Updating trail intensity}

After all ants have constructed their solutions, the trail intensities are updated using the solution of $\gamma$ elitist ants. The elitist ants are defined as the $\gamma$ best ant solution found so far. Elitist ants are updated by comparing 
the present elitist ant solutions with the current ant solutions. While updating elitist ants, the algorithm ensures that the solutions of elitist ants are distinct from each other. The trail intensity of assigning task $T_{i}$ to resource $R_{k}$ is updated using elitist ants as follows:

$$
\tau_{i k}^{\text {new }}=\rho \times \tau_{i k}^{\text {old }}+\sum_{\mu=1}^{\gamma} \Delta \tau_{i k}^{\mu}, i=1,2, \ldots, n t ; k=1,2, \ldots, m
$$

Here $\rho$ is the called evaporation factor, taking value between 0 and 1 . The first term in equation (24) represents evaporations of existing trail intensity. The second term represents the deposition of pheromone by $\gamma$ elitist ant where

$$
\Delta \tau_{i k}^{\mu}= \begin{cases}1 / L^{\mu} & \text { if task } T_{i} \text { is assigned to resouce } R_{k} \text { in the } \mu^{\text {th }} \text { eltist ant } \\ 0 & \text { otherwise }\end{cases}
$$

Here $L^{\mu}$ is the objective function value for the $\mu^{\text {th }}$ elitist ant solution. Our ACS algorithm has a computation complexity of $\mathrm{O}\left(\mathrm{n}^{\wedge} 2\right)$, much better than the prior work baselines with complexity of $\mathrm{O}\left(\mathrm{n}^{\wedge} 2 *\right.$ $\log (n))$.

\section{Performance Evaluation}

This section presents numerical experiment and evaluation of proposed algorithms. The optimal solution for small problem instances is obtained by solving MILP formulation. 'A Mathematical Programming Language' (AMPL) software with CPLEX solver is used to solve the MILP formulation. The software can solve the problem size of 10 subtasks within 15 minutes. In this section, we provide the experiment for small instances as well as the large problem instances. All the algorithms (i.e., heuristics and meta-heuristic) are implemented in same simulation environment, coded in C Language and run on an AMD Opteron $2.6 \mathrm{GHz}$ PC with 16 GB memory on Unix OS. The HH and SS heuristic are coded on the basis of pseudo code available in those papers. We used the following notations for reporting results.

HH: HH algorithm of Herrmann et al., (1997)

SS: $\quad$ SS algorithm of Liu \& Yang (2011) 
EST: $\quad$ EST based proposed heuristic algorithm

ACS: Ant colony algorithm based solution

MKS: Makespan value produced by an algorithm

TCT: Total completion time value produced by an algorithm

RPD: Relative percentage deviation of an algorithm from the best solution

CPU: CPU time (in seconds) consumed by an algorithm

PG: Percentage gap of an algorithm form the optimal solution

$\mathrm{n}$ : Independent tasks received by a cloud computing service provider at a particular time

$\mathrm{m}$ : number of virtual machine/commodity computers available to process above $\mathrm{n}$ at a particular time

nt: total number of inter-dependent subtasks of $n$ tasks

The performance of proposed solution method is evaluated through PG and RPD value. The formula used to calculate the PG and RPD is given below.

$\mathrm{PG}=\left\{(A S-O p t)^{*} 100\right\} / O p t$

$\mathrm{RPD}=\{(A S-$ Best $) * 100\} /$ Best

Where, AS represents the solution of the algorithm, Opt represents the optimal solution and Best represents the best solution among all the solutions used for evaluation.

\subsection{Experiments on Small Instances}

The small instances are generated to find the optimal solution. In small instances, the number of tasks considered is 2, 3, 4 and 5 and the numbers of resources considered are 2, 3 and 4. Thus, a total of 12 groups of problem instances are generated. These groups are represented by AY1 to AY12. We generated 10 problem instances for each group and thus the total of 120 small problem instances is generated. The number of subtasks for each task are generated from a uniform distribution in the range of $[2,3]$. The process time of tasks are generated from a uniform distribution in the range of [10, 25].

\subsubsection{Experiments on small instances for total completion time objective}

The results of HH heuristic, SS heuristics and EST heuristics, and Ant colony algorithm for small instances are reported in Table 3 for the total completion time objective. The average total completion time obtained 
for HH heuristic, SS heuristics, EST heuristics and ACS meta-heuristic are shown in Table 3 along with their Percentage Gap (PG) value. The average percentage gap for 12 groups of problem instances are reported in the bottom of the Table 3. The average PG of HH and SS heuristic from optimal solution is noted as $10.7 \%$ and $4.26 \%$ respectively. The results show that the solution is far away from the optimal solution for existing heuristics. The average PG of EST heuristic is noted as $2.46 \%$, which is good as compare to the existing $\mathrm{HH}$ heuristic and SS heuristic. The PG from the optimal solution for ACS is noted as $0.29 \%$ which is close to the optimal solution. Moreover, ACS metaheuristic gives the best results for all 120 small instances problem as compare to HH heuristic, SS heuristics and EST heuristics.

The CPU time of HH heuristic, SS heuristics and EST heuristics and ACS meta-heuristic is also reported in Table 3. The comparison of CPU is fair because all the algorithms are executed on the same simulation environment. The CPU time of optimal method is on average 1079.91 seconds for 120 problem instances. It can be observed that the CPU time for optimal method increases exponentially with increase in problem size. The exponential increase in CPU time makes the use of optimal method practically impossible for solving bigger problem instances. This observation also justifies the use of EST heuristic and ACS metaheuristic over HH heuristic and SS heuristics for solving the task scheduling problem.

Table 3: Experimental results of small instances for total completion time objective

\begin{tabular}{|c|c|c|c|c|c|c|c|c|c|c|c|c|c|c|c|c|c|}
\hline \multirow{2}{*}{$\begin{array}{c}\text { Instance } \\
\text { No. }\end{array}$} & \multirow{2}{*}{$n$} & \multirow{2}{*}{$m$} & \multirow{2}{*}{$n t$} & \multicolumn{2}{|c|}{ Optimal Solution } & \multicolumn{3}{|c|}{ HH Heuristic } & \multicolumn{3}{|c|}{ SS Heuristic } & \multicolumn{3}{|c|}{ EST Heuristic } & \multicolumn{3}{|c|}{ ACS } \\
\hline & & & & TCT & $\mathrm{CPU}$ & TCT & $\mathrm{CPU}$ & PG & TCT & $\mathrm{CPU}$ & PG & TCT & CPU & PG & TCT & CPU & PG \\
\hline AY1 & 2 & 2 & 6 & 319.2 & 0.2 & 337.5 & $<1$ & 5.34 & 328.80 & $<1$ & 2.95 & 322.40 & $<1$ & 0.86 & 320.40 & $<1$ & 0.41 \\
\hline AY 2 & 2 & 3 & 5 & 212.30 & 0.18 & 222.30 & $<1$ & 5.76 & 217.80 & $<1$ & 2.44 & 217.90 & $<1$ & 2.39 & 213.30 & $<1$ & 0.43 \\
\hline AY 3 & 2 & 4 & 5 & 138.60 & 0.21 & 141.20 & $<1$ & 1.80 & 148.80 & $<1$ & 7.43 & 141.10 & $<1$ & 1.29 & 139.50 & $<1$ & 0.97 \\
\hline AY4 & 3 & 2 & 8 & 431.00 & 4.47 & 474.9 & $<1$ & 10.96 & 454.4 & $<1$ & 6.46 & 450.1 & $<1$ & 4.77 & 432 & $<1$ & 0.35 \\
\hline AY5 & 3 & 3 & 8 & 330.30 & 6.01 & 376.20 & $<1$ & 14.13 & 344.30 & $<1$ & 4.02 & 337.30 & $<1$ & 2.16 & 331.10 & $<1$ & 0.27 \\
\hline AY6 & 3 & 4 & 8 & 316.30 & 11.34 & 335 & $<1$ & 5.80 & 337 & $<1$ & 6.39 & 323.5 & $<1$ & 2.20 & 316.3 & $<1$ & 0 \\
\hline AY 7 & 4 & 2 & 10 & 732.40 & 37.47 & 830.7 & $<1$ & 15.57 & 752.6 & $<1$ & 3.17 & 748.6 & $<1$ & 2.46 & 733.3 & $<1$ & 0.14 \\
\hline AY 8 & 4 & 3 & 10 & 611.80 & 123.92 & 686.7 & $<1$ & 12.62 & 631.1 & $<1$ & 3.52 & 631.4 & $<1$ & 3.52 & 612.7 & $<1$ & 0.16 \\
\hline AY 9 & 4 & 4 & 10 & 416.50 & 78.80 & 462.1 & $<1$ & 11.38 & 436 & $<1$ & 4.71 & 432.9 & $<1$ & 3.88 & 417 & $<1$ & 0.13 \\
\hline AY10 & 5 & 2 & 12 & 1173.10 & 1582.59 & 1345.7 & $<1$ & 15.85 & 1202 & $<1$ & 2.48 & 1183.5 & $<1$ & 0.88 & 1174.7 & $<1$ & 0.10 \\
\hline AY11 & 5 & 3 & 12 & 848.30 & 3348.49 & 967.3 & $<1$ & 14.87 & 870.4 & $<1$ & 2.79 & 865 & $<1$ & 2.26 & 850.6 & $<1$ & 0.22 \\
\hline AY 12 & 5 & 4 & 13 & 667.70 & 7765.25 & 761.8 & $<1$ & 14.76 & 700.20 & $<1$ & 4.95 & 685.6 & $<1$ & 2.64 & 670 & $<1$ & 0.32 \\
\hline \multicolumn{4}{|c|}{ Average } & 516.46 & 1079.91 & 578.45 & $<1$ & 10.74 & 535.28 & $<1$ & 4.28 & 528.28 & $<1$ & 2.44 & 517.58 & $<1$ & 0.29 \\
\hline
\end{tabular}


Figure 5 depicts that the performance of ACS is better than the performance of HH, SS and EST heuristic. ACS obtains nearby results to optimal solution for total completion time objective.

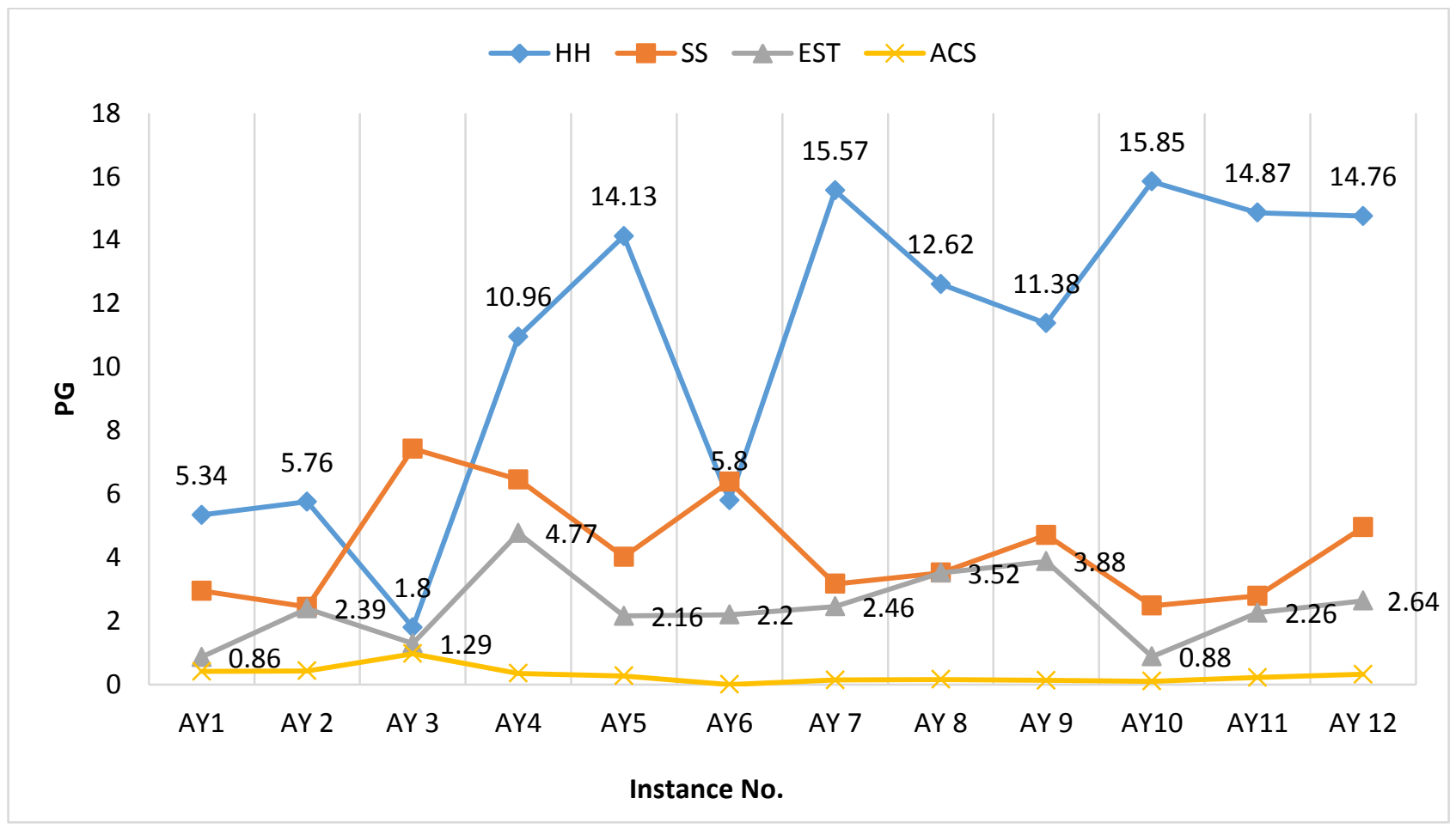

Figure 5: Percentage Gap of Algorithms with TCT objective function for small data set.

\subsubsection{Experiments on small instances for Makespan objective}

The performance results of HH, SS, EST and ACS for Makespan objective problem for small instances are reported in Table 4 and Figure 6. Table 4 reports the RPD value of HH heuristics, SS heuristics, EST heuristics and ACS meta-heuristic viz. $10.03 \%, 13.12 \%, 9.19 \%$ and 1.31 respectively. The PG value of $\mathrm{HH}$ heuristics, SS heuristics and EST heuristics is far away from the optimal solution for Makespan objective. The PG from the optimal solution for ACS is noted as $1.37 \%$, which is very near to the optimal solution.

Table 4: Experiment results of small instances for Makespan objective problem

\begin{tabular}{|c|c|c|c|c|c|c|c|c|c|c|c|c|c|c|c|c|c|}
\hline \multirow{2}{*}{$\begin{array}{c}\text { Instance } \\
\text { no. }\end{array}$} & \multirow{2}{*}{$n$} & \multirow[t]{2}{*}{$m$} & \multirow{2}{*}{$n t$} & \multicolumn{2}{|c|}{$\begin{array}{l}\text { Optimal } \\
\text { Solution }\end{array}$} & \multicolumn{3}{|c|}{ HH Heuristic } & \multicolumn{3}{|c|}{ SS Heuristic } & \multicolumn{3}{|c|}{ EST Heuristic } & \multicolumn{3}{|c|}{ ACS Heuristic } \\
\hline & & & & MKS & $\mathrm{CPU}$ & MKS & $\mathrm{CPU}$ & PG & MKS & $\mathrm{CPU}$ & PG & MKS & $\mathrm{CPU}$ & PG & MKS & $\mathrm{CPU}$ & PG \\
\hline AY1 & 2 & 2 & 6 & 77 & 0.19 & 78.6 & $<1$ & 1.95 & 82 & $<1$ & 6.64 & 80.8 & $<1$ & 4.41 & 77 & $<1$ & 0 \\
\hline AY 2 & 2 & 3 & 5 & 55.8 & 0.16 & 57.5 & $<1$ & 3.03 & 60.1 & $<1$ & 7.39 & 60.3 & $<1$ & 7.32 & 56 & $<1$ & 0.31 \\
\hline AY 3 & 2 & 4 & 5 & 39 & 0.10 & 39.8 & $<1$ & 2.09 & 43 & $<1$ & 10.29 & 40.2 & $<1$ & 2.46 & 39.5 & $<1$ & 1.14 \\
\hline AY4 & 3 & 2 & 8 & 82.1 & 0.62 & 90.6 & $<1$ & 10.29 & 93.9 & $<1$ & 16.39 & 92.5 & $<1$ & 11.34 & 82.9 & $<1$ & 0.97 \\
\hline
\end{tabular}




\begin{tabular}{|c|c|c|c|c|c|c|c|c|c|c|c|c|c|c|c|c|c|}
\hline AY5 & 3 & 3 & 8 & 60.3 & 0.59 & 66.8 & $<1$ & 10.92 & 68.6 & $<1$ & 13.95 & 66.6 & $<1$ & 9.18 & 61.1 & $<1$ & 1.27 \\
\hline AY6 & 3 & 4 & 8 & 55.4 & 0.77 & 57.3 & $<1$ & 3.44 & 64.4 & $<1$ & 15.37 & 60.9 & $<1$ & 8.09 & 55.6 & $<1$ & 0.40 \\
\hline AY 7 & 4 & 2 & 10 & 110 & 3.02 & 124.4 & $<1$ & 14.10 & 122.2 & $<1$ & 11.74 & 120.1 & $<1$ & 8.61 & 111.4 & $<1$ & 1.17 \\
\hline AY 8 & 4 & 3 & 10 & 81.1 & 4.03 & 91.3 & $<1$ & 12.87 & 92.4 & $<1$ & 14.11 & 92.7 & $<1$ & 12.11 & 82.1 & $<1$ & 1.09 \\
\hline AY 9 & 4 & 4 & 10 & 59 & 3.01 & 65.8 & $<1$ & 12.50 & 68.9 & $<1$ & 17.60 & 69.3 & $<1$ & 14.48 & 60.3 & $<1$ & 2.23 \\
\hline AY10 & 5 & 2 & 12 & 140.9 & 11.44 & 158.5 & $<1$ & 13.03 & 156.2 & $<1$ & 11.17 & 149.1 & $<1$ & 5.39 & 142.3 & $<1$ & 0.86 \\
\hline AY11 & 5 & 3 & 12 & 94.4 & 18.64 & 110.2 & $<1$ & 16.74 & 104.9 & $<1$ & 11.13 & 105.7 & $<1$ & 10.77 & 96.6 & $<1$ & 2.22 \\
\hline AY 12 & 5 & 4 & 13 & 72.2 & 20.72 & 85.8 & $<1$ & 19.40 & 87.6 & $<1$ & 21.69 & 86.4 & $<1$ & 16.16 & 75.3 & $<1$ & 4.02 \\
\hline \multicolumn{4}{|c|}{ Average } & 77 & 5.27 & 85.55 & $<1$ & 10 & 87 & $<1$ & 13.1 & 85 & $<1$ & 9.2 & 78 & $<1$ & 1.3 \\
\hline
\end{tabular}

Figure 5 depicts that the performance of metaheuristic (ACS) is better than the HH, SS and EST. ACS generates the solution close to the optimal solution for Makespan objective. An interesting observation about the CPU time of optimal solution can be made from Table 3 and Table 4 . The CPU time of Makespan objective is considerably lower than the CPU time of the total completion time objective. The results indicate that solving Makespan objective problem is easier than solving the total completion time objective problem.

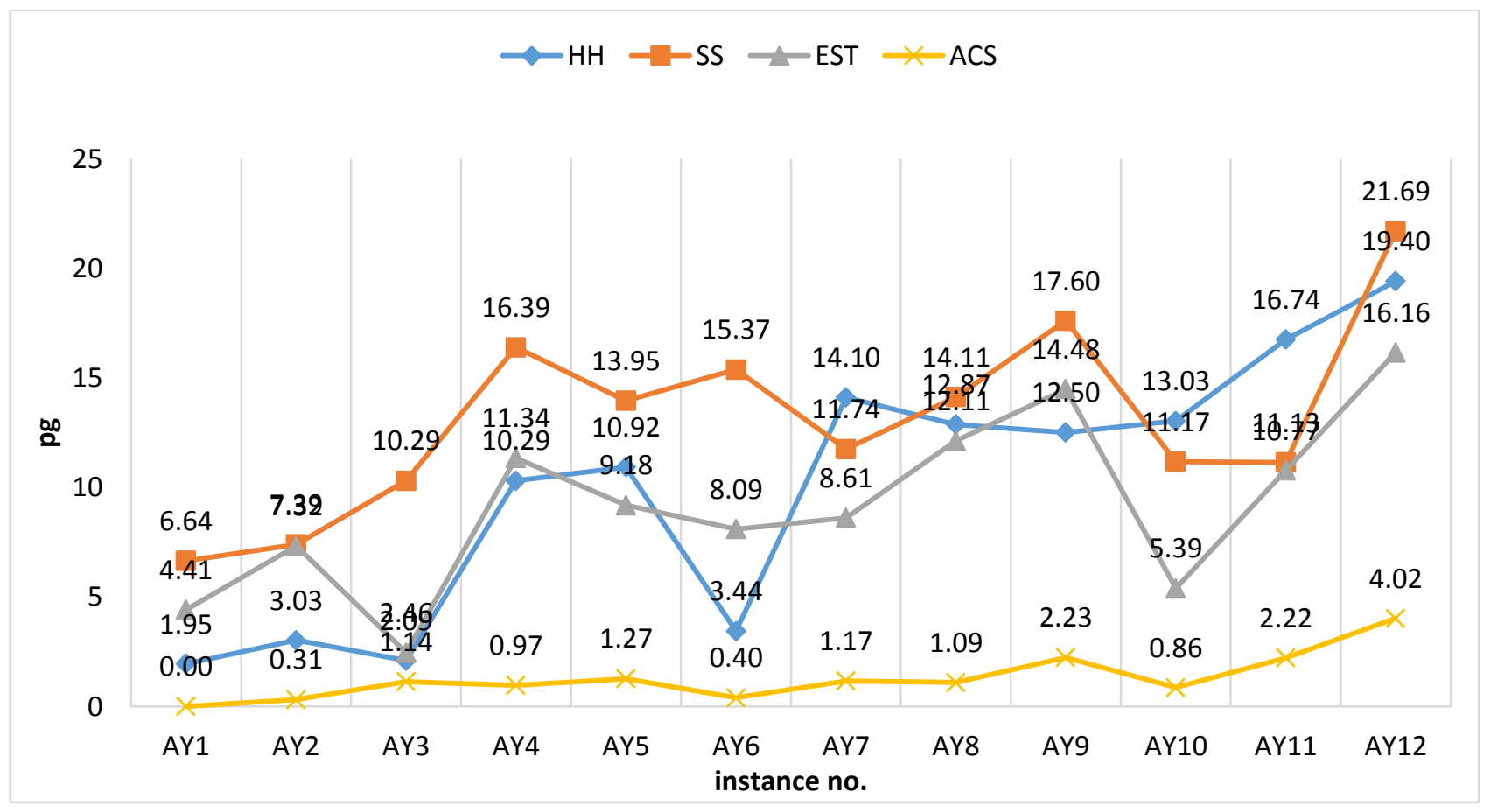

Figure 6: Percentage Gap of Algorithms with Makespan objective function for small data set

\subsection{Experiment results of large instance}

This section provides the numerical analysis for large problem instances. The number of tasks $(n)$ considered are $15,30,45,60,75,90,105,120,135$ and 150 and the number of resources $(m)$ considered is 2,5 and 7. 
Thus, the total of 30 groups of problem instances is generated. In a given problem instance, the number of subtasks for a given task is generated in the range of $[2,15]$. The process time of subtasks are generated from a uniform distribution in the range of $[10,25]$.

\subsubsection{Experimental results on large instances for total completion time objective}

Table 5 reports the experimental results of total completion time objective for the large problem instances.

Table 5: Numerical results for heuristics and meta-heuristics for total completion time objective problem

\begin{tabular}{|c|c|c|c|c|c|c|c|c|c|c|c|c|c|c|c|}
\hline \multirow{2}{*}{$\begin{array}{c}\text { Instance } \\
\text { No. }\end{array}$} & \multirow{2}{*}{$n$} & \multirow{2}{*}{$m$} & \multirow{2}{*}{$n t$} & \multicolumn{3}{|c|}{ HH Heuristic } & \multicolumn{3}{|c|}{ SS Heuristic } & \multicolumn{3}{|c|}{ EST Heuristic } & \multicolumn{3}{|c|}{$\mathrm{ACS}$} \\
\hline & & & & TCT & $\mathrm{CPU}$ & RPD & TCT & $\mathrm{CPU}$ & RPD & TCT & $\mathrm{CPU}$ & RPD & TCT & $\mathrm{CPU}$ & RPD \\
\hline BG1 & 15 & 2 & 51 & 23234.7 & $<1$ & 16.62 & 20378.2 & $<1$ & 1.04 & 20349.5 & $<1$ & 0.93 & 20171.30 & 0.70 & 0 \\
\hline BG2 & 15 & 5 & 49 & 8141.1 & $<1$ & 16.39 & 7149.4 & $<1$ & 1.78 & 7102.0 & $<1$ & 1.15 & 7023.20 & 1.10 & 0 \\
\hline BG3 & 15 & 7 & 54 & 7312.5 & $<1$ & 18.24 & 6285.4 & $<1$ & 1.69 & 6245.2 & $<1$ & 1.08 & 6180.30 & 1.70 & 0 \\
\hline BG4 & 30 & 2 & 103 & 92248.5 & $<1$ & 16.42 & 80250.5 & $<1$ & 0.94 & 80165.9 & $<1$ & 0.83 & 79531.90 & 10.50 & 0 \\
\hline BG5 & 30 & 5 & 101 & 33339.9 & $<1$ & 22.33 & 27646.0 & $<1$ & 0.93 & 27554.1 & $<1$ & 0.61 & 27399.90 & 13.60 & 0 \\
\hline BG6 & 30 & 7 & 106 & 27342.9 & $<1$ & 19.66 & 23123.7 & $<1$ & 1.08 & 23009.6 & $<1$ & 0.54 & 22883.00 & 18.50 & 0 \\
\hline BG7 & 45 & 2 & 152 & 210796.4 & $<1$ & 18.25 & 181339.4 & $<1$ & 0.81 & 181030.5 & $<1$ & 0.61 & 179964.00 & 41.40 & 0 \\
\hline BG8 & 45 & 5 & 150 & 80986.1 & $<1$ & 21.52 & 67609.4 & $<1$ & 0.60 & 67447.2 & $<1$ & 0.37 & 67213.70 & 49.00 & 0 \\
\hline BG9 & 45 & 7 & 153 & 57569.0 & $<1$ & 20.06 & 48784.9 & $<1$ & 0.78 & 48541.1 & $<1$ & 0.25 & 48424.60 & 58.10 & 0 \\
\hline BG10 & 60 & 2 & 205 & 359178.1 & $<1$ & 20.09 & 303241.2 & $<1$ & 0.82 & 302909.2 & $<1$ & 0.70 & 300843.50 & 106.90 & 0 \\
\hline BG11 & 60 & 5 & 205 & 141010.1 & $<1$ & 20.16 & 118385.3 & $<1$ & 0.57 & 118197.8 & $<1$ & 0.40 & 117731.20 & 134.60 & 0 \\
\hline BG12 & 60 & 7 & 206 & 101209.1 & $<1$ & 21.05 & 84537.5 & $<1$ & 0.61 & 84246.8 & $<1$ & 0.27 & 84034.90 & 152.70 & 0 \\
\hline BG13 & 75 & 2 & 254 & 684446.9 & $<1$ & 15.57 & 598368.9 & $<1$ & 0.55 & 597737.5 & $<1$ & 0.44 & 595122.00 & 217.20 & 0 \\
\hline BG14 & 75 & 5 & 257 & 232465.4 & $<1$ & 22.21 & 193354.5 & $<1$ & 0.47 & 192926.7 & $<1$ & 0.24 & 192495.50 & 281.70 & 0 \\
\hline BG15 & 75 & 7 & 257 & 153139.5 & $<1$ & 21.83 & 127408.5 & $<1$ & 0.56 & 127013.2 & $<1$ & 0.24 & 126709.30 & 319.70 & 0 \\
\hline BG16 & 90 & 2 & 303 & 804813.9 & $<1$ & 19.95 & 681828.7 & $<1$ & 0.73 & 680945.6 & $<1$ & 0.59 & 676930.60 & 379.90 & 0 \\
\hline BG17 & 90 & 5 & 300 & 300210.3 & $<1$ & 22.76 & 247651.6 & $<1$ & 0.50 & 247176.3 & $<1$ & 0.29 & 246482.80 & 467.10 & 0 \\
\hline BG18 & 90 & 7 & 305 & 215329.1 & $<1$ & 20.86 & 179634.3 & $<1$ & 0.47 & 179158.3 & $<1$ & 0.20 & 178809.40 & 579.20 & 0 \\
\hline BG19 & 105 & 2 & 367 & 1250548.4 & $<1$ & 17.49 & 1075103.5 & $<1$ & 0.62 & 1073774.6 & $<1$ & 0.49 & 1068609.60 & 689.70 & 0 \\
\hline BG20 & 105 & 5 & 360 & 411214.1 & $<1$ & 24.53 & 333454.4 & $<1$ & 0.44 & 333115.2 & $<1$ & 0.33 & 332017.50 & 846.80 & 0 \\
\hline BG21 & 105 & 7 & 360 & 309928.5 & $<1$ & 20.40 & 258690.6 & $<1$ & 0.45 & 257913.3 & $<1$ & 0.14 & 257550.20 & 962.10 & 0 \\
\hline BG22 & 120 & 2 & 405 & 1456331.2 & $<1$ & 19.49 & 1235319.1 & $<1$ & 0.63 & 1234693.6 & $<1$ & 0.56 & 1228088.40 & 972.90 & 0 \\
\hline BG23 & 120 & 5 & 407 & 565726.7 & $<1$ & 22.46 & 465574.8 & $<1$ & 0.46 & 464651.3 & $<1$ & 0.26 & 463463.00 & 1245.40 & 0 \\
\hline BG24 & 120 & 7 & 409 & 415008.3 & $<1$ & 20.55 & 347439.6 & $<1$ & 0.42 & 346619.2 & $<1$ & 0.16 & 346069.00 & 1452.60 & 0 \\
\hline BG25 & 135 & 2 & 459 & 1898078.2 & $<1$ & 20.70 & 1612879.8 & $<1$ & 0.58 & 1611788.4 & $<1$ & 0.51 & 1604489.00 & 1538.40 & 0 \\
\hline BG26 & 135 & 5 & 459 & 736659.2 & $<1$ & 22.02 & 608457.3 & $<1$ & 0.43 & 607255.0 & $<1$ & 0.23 & 605897.90 & 1937.60 & 0 \\
\hline BG27 & 135 & 7 & 461 & 519861.5 & $<1$ & 22.14 & 429560.2 & $<1$ & 0.41 & 428478.9 & $<1$ & 0.14 & 427871.40 & 2175.90 & 0 \\
\hline BG28 & 150 & 2 & 508 & 2285765.6 & $<1$ & 20.39 & 1929552.9 & $<1$ & 0.57 & 1928130.1 & $<1$ & 0.48 & 1919198.30 & 2171.40 & 0 \\
\hline BG29 & 150 & 5 & 509 & 880779.3 & $<1$ & 23.09 & 723195.0 & $<1$ & 0.42 & 721789.1 & $<1$ & 0.23 & 720166.60 & 2635.40 & 0 \\
\hline BG30 & 150 & 7 & 515 & 643509.3 & $<1$ & 20.87 & 536226.5 & $<1$ & 0.34 & 535015.5 & $<1$ & 0.12 & 534410.90 & 2984.40 & 0 \\
\hline Average & 83 & 5 & 281 & 496872.8 & $<1$ & 20.3 & 418414.4 & $<1$ & 0.69 & 417832.7 & $<1$ & 0.45 & 416192.76 & 748.21 & 0 \\
\hline
\end{tabular}


The average RPD value over 300 problem instances indicate that the ACS has the best performance followed by EST heuristic, SS heuristic and then by HH heuristic. The average RPD of ACS, EST heuristic, SS heuristic and $\mathrm{HH}$ heuristic are $0 \%, 0.45 \%, 0.69 \%$ and $20.3 \%$ respectively.

Figure 7 visualizes the RPD value of all four algorithms for 30 problem groups. The performance of ACS is better than the performance of other algorithms. The performance of $\mathrm{HH}$ heuristic is poor, which is indicated in small problem instances as well. One of the reasons for poor performance of the heuristic is that it did not use any list algorithm property of the problem. The performance of SS heuristic is close to the proposed EST heuristic but still inferior than the EST heuristic. The SS heuristic uses earliest available machine assignment rule. We have shown in Section 4 that the earliest machine assignment rule does not provide dominant solution of the problem. This is one of the reason for the poor perforamnce of SS heuristic as compared to the EST heuristic.

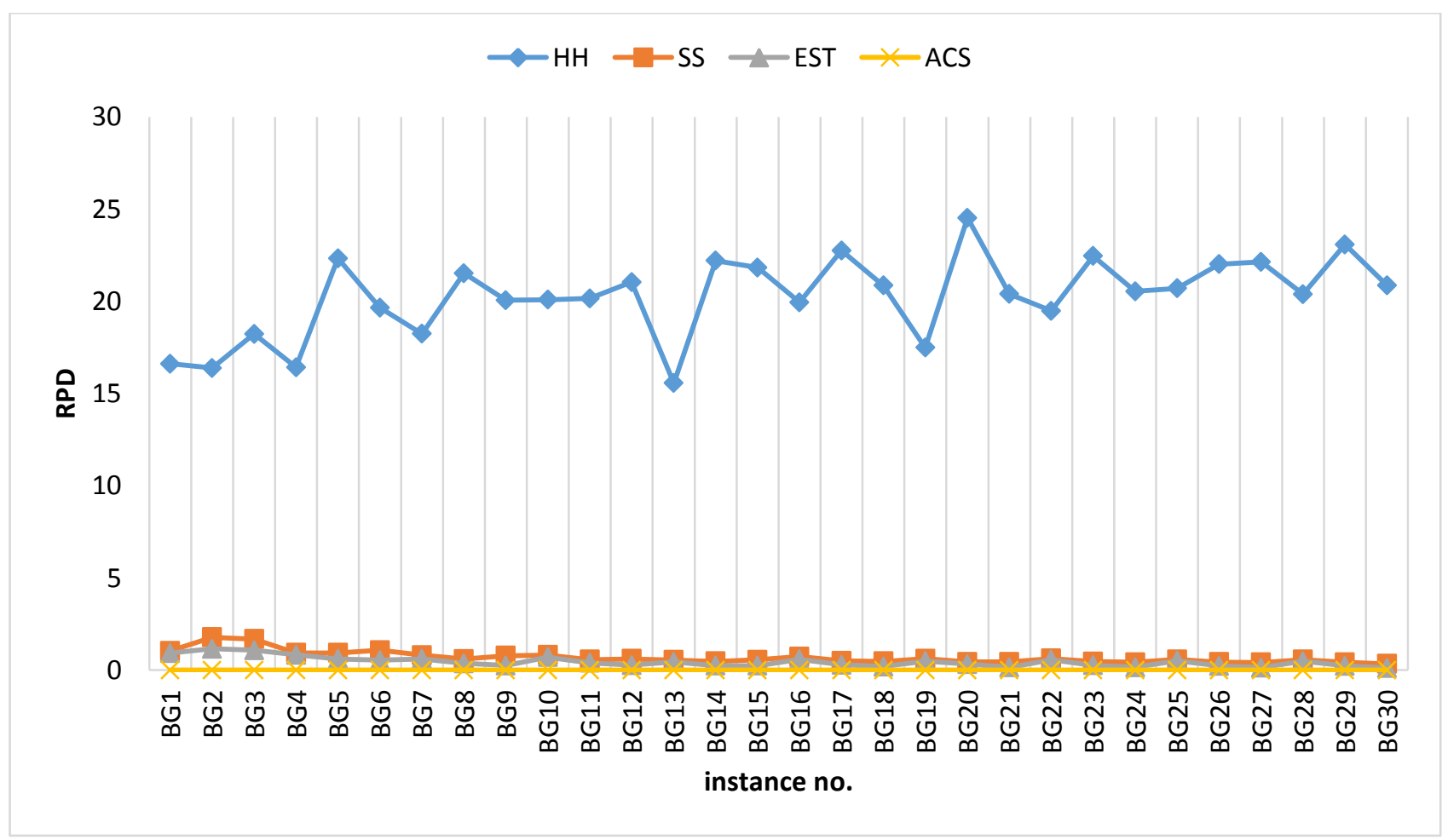

Figure 7: RPD of Algorithms with TCT objective function for large data set.

Figure 8 shows the comparison of scheduling time for algorithms with small dataset and ACS performs better than EST, SS and HH. ACS has 13\%, $15.5 \%$ and $21 \%$ less than EST, SS and HH respectively. The reason 
behind better performance of ACS is the implementation of precedence task constraints during task scheduling.

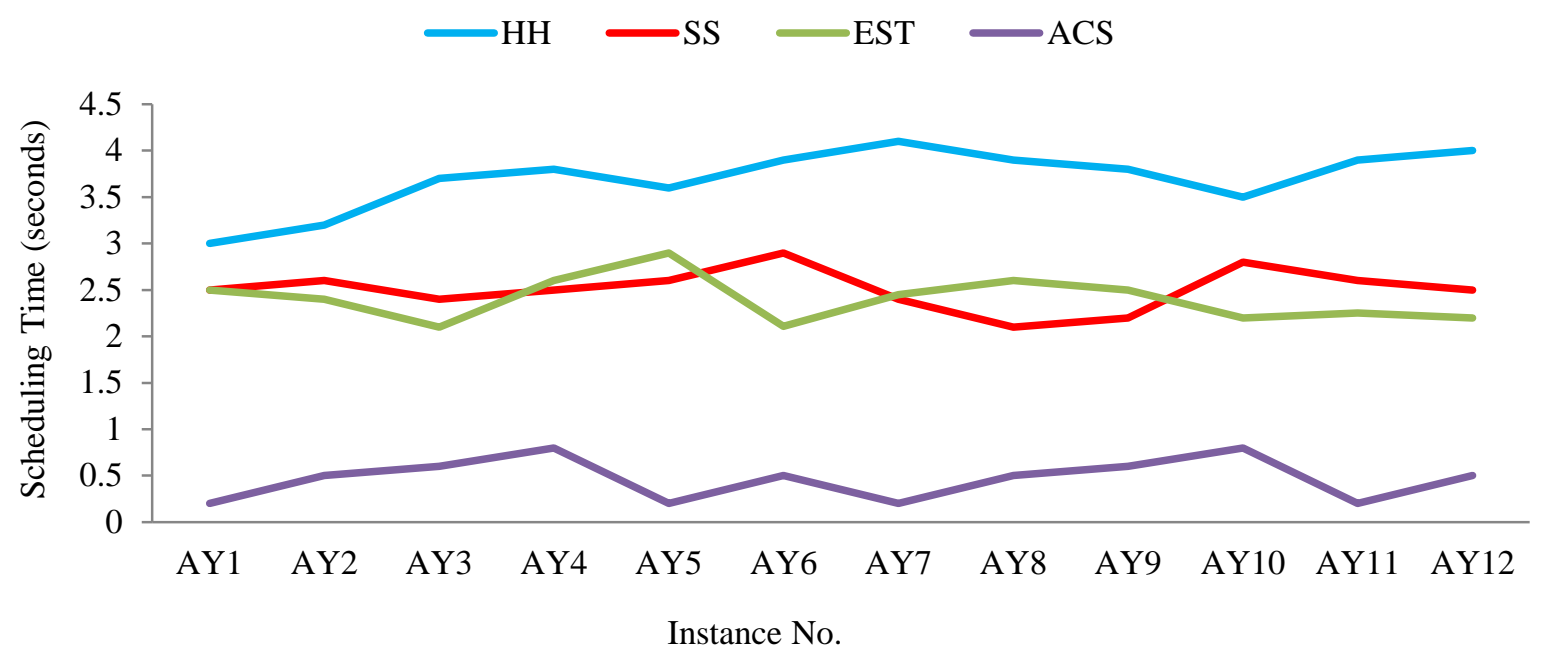

Figure 8: Comparison of scheduling time for algorithms with small dataset

\subsubsection{Experimental results on large instances for Makespan objective problem}

Table 6 and Figure 9 report the performace of HH, SS, EST \& ACS in large problem instances for Makespan objective.

Table: 6 Experiment results of large instance for Makespan objective

\begin{tabular}{|c|c|c|c|c|c|c|c|c|c|c|c|c|c|c|c|}
\hline \multirow{2}{*}{$\begin{array}{c}\text { Job } \\
\text { Instance }\end{array}$} & \multirow{2}{*}{$n$} & \multirow{2}{*}{$m$} & \multirow{2}{*}{$n t$} & \multicolumn{3}{|c|}{ HH Heuristic } & \multicolumn{3}{|c|}{ SS Heuristic } & \multicolumn{3}{|c|}{ EST Heuristic } & \multicolumn{3}{|c|}{ ACS } \\
\hline & & & & MKS & $\mathrm{CPU}$ & RPD & MKS & $\mathrm{CPU}$ & RPD & MKS & $\mathrm{CPU}$ & RPD & MKS & CPU & RPD \\
\hline BG1 & 15 & 2 & 51 & 671.1 & $<1$ & 13.4 & 613.30 & $<1$ & 3.49 & 608.50 & $<1$ & 2.69 & 593.10 & $<1$ & 0.0 \\
\hline BG2 & 15 & 5 & 49 & 242.30 & $<1$ & 13.7 & 235.20 & $<1$ & 10.07 & 229.80 & $<1$ & 7.88 & 213.40 & 0.80 & 0.0 \\
\hline BG3 & 15 & 7 & 54 & 200.8 & $<1$ & 17.5 & 188.20 & $<1$ & 10.35 & 186.20 & $<1$ & 9.28 & 170.80 & 1.20 & 0.0 \\
\hline BG4 & 30 & 2 & 103 & 1366.5 & $<1$ & 13.2 & 1238.40 & $<1$ & 2.43 & 1229.80 & $<1$ & 1.67 & 1209.20 & 4.00 & 0.0 \\
\hline BG5 & 30 & 5 & 101 & 501.3 & $<1$ & 18.9 & 441.10 & $<1$ & 4.26 & 440.40 & $<1$ & 4.25 & 423.30 & 7.80 & 0.0 \\
\hline BG6 & 30 & 7 & 106 & 386.1 & $<1$ & 15.6 & 353.4 & $<1$ & 5.74 & 353.5 & $<1$ & 5.64 & 334.30 & 10.4 & 0.0 \\
\hline BG7 & 45 & 2 & 152 & 2086.8 & $<1$ & 15.3 & 1845.9 & $<1$ & 1.70 & 1838.1 & $<1$ & 1.20 & 1815.40 & 13.2 & 0.0 \\
\hline BG8 & 45 & 5 & 150 & 796.9 & $<1$ & 18.8 & 692.6 & $<1$ & 3.01 & 690.4 & $<1$ & 2.68 & 672.40 & 24.3 & 0.0 \\
\hline BG9 & 45 & 7 & 153 & 551.3 & $<1$ & 16.6 & 490.8 & $<1$ & 3.74 & 483.5 & $<1$ & 2.15 & 473.50 & 27.6 & 0.0 \\
\hline BG10 & 60 & 2 & 205 & 2735.6 & $<1$ & 16.7 & 2391.7 & $<1$ & 1.80 & 2382.3 & $<1$ & 1.40 & 2349.80 & 31.4 & 0.0 \\
\hline BG11 & 60 & 5 & 205 & 1036.7 & $<1$ & 17.3 & 904.9 & $<1$ & 2.43 & 907 & $<1$ & 2.57 & 884.40 & 58.4 & 0.0 \\
\hline BG12 & 60 & 7 & 206 & 724.6 & $<1$ & 16.8 & 642.4 & $<1$ & 3.36 & 634.3 & $<1$ & 2.18 & 621.40 & 67.3 & 0.0 \\
\hline BG13 & 75 & 2 & 254 & 3876 & $<1$ & 14.1 & 3439.3 & $<1$ & 1.14 & 3428.6 & $<1$ & 0.80 & 3401.80 & 58.8 & 0.0 \\
\hline BG14 & 75 & 5 & 257 & 1332.9 & $<1$ & 19.5 & 1143.4 & $<1$ & 2.19 & 1136.6 & $<1$ & 1.54 & 1119.40 & 118.9 & 0.0 \\
\hline BG15 & 75 & 7 & 257 & 892.7 & $<1$ & 18.2 & 781 & $<1$ & 3.16 & 774.7 & $<1$ & 2.38 & 757.50 & 119.7 & 0.0 \\
\hline
\end{tabular}




\begin{tabular}{|c|c|c|c|c|c|c|c|c|c|c|c|c|c|c|c|}
\hline BG16 & 90 & 2 & 303 & 4105.4 & $<1$ & 16.6 & 3580.2 & $<1$ & 1.20 & 3572.9 & $<1$ & 1.01 & 3537.90 & 99.2 & 0.0 \\
\hline BG17 & 90 & 5 & 300 & 1515.5 & $<1$ & 19.3 & 1298.1 & $<1$ & 1.98 & 1290.4 & $<1$ & 1.35 & 1273.70 & 173.4 & 0.0 \\
\hline BG18 & 90 & 7 & 305 & 1055 & $<1$ & 17.9 & 915.7 & $<1$ & 2.27 & 911.4 & $<1$ & 1.82 & 895.60 & 198.4 & 0.0 \\
\hline BG19 & 105 & 2 & 367 & 5188.7 & $<1$ & 14.9 & 4577.2 & $<1$ & 1.22 & 4568.3 & $<1$ & 1.03 & 4522.20 & 167.5 & 0.0 \\
\hline BG20 & 105 & 5 & 360 & 1762.3 & $<1$ & 19.3 & 1503.8 & $<1$ & 1.60 & 1498.7 & $<1$ & 1.22 & 1480.60 & 298.5 & 0.0 \\
\hline BG21 & 105 & 7 & 360 & 1281 & $<1$ & 17.7 & 1106.2 & $<1$ & 1.53 & 1102.9 & $<1$ & 1.21 & 1089.40 & 302.4 & 0.0 \\
\hline BG22 & 120 & 2 & 405 & 5551.9 & $<1$ & 16.1 & 4850.2 & $<1$ & 1.16 & 4844.3 & $<1$ & 1.02 & 4796.60 & 226.4 & 0.0 \\
\hline BG23 & 120 & 5 & 407 & 2098.2 & $<1$ & 20.5 & 1762.1 & $<1$ & 1.08 & 1762.9 & $<1$ & 1.13 & 1743.40 & 417 & 0.0 \\
\hline BG24 & 120 & 7 & 409 & 1483.4 & $<1$ & 17.8 & 1284.8 & $<1$ & 1.82 & 1280.4 & $<1$ & 1.42 & 1262.30 & 460.4 & 0.0 \\
\hline BG25 & 135 & 2 & 459 & 6292.6 & $<1$ & 16.7 & 5489.2 & $<1$ & 1.08 & 5476.6 & $<1$ & 0.85 & 5431.50 & 334.6 & 0.0 \\
\hline BG26 & 135 & 5 & 459 & 2395.3 & $<1$ & 18.6 & 2043.8 & $<1$ & 1.18 & 2034.1 & $<1$ & 0.69 & 2020.40 & 602.4 & 0.0 \\
\hline BG27 & 135 & 7 & 461 & 1668 & $<1$ & 18.5 & 1425.7 & $<1$ & 1.08 & 1423.9 & $<1$ & 0.92 & 1411.10 & 629.7 & 0.0 \\
\hline BG28 & 150 & 2 & 508 & 6937 & $<1$ & 17.0 & 6020.8 & $<1$ & 1.09 & 6007.4 & $<1$ & 0.87 & 5956.60 & 453.9 & 0.0 \\
\hline BG29 & 150 & 5 & 509 & 2602.4 & $<1$ & 19.4 & 2211 & $<1$ & 1.18 & 2204.6 & $<1$ & 0.90 & 2184.60 & 840.8 & 0.0 \\
\hline BG30 & 150 & 7 & 515 & 1849.4 & $<1$ & 18.1 & 1590.2 & $<1$ & 1.38 & 1581.7 & $<1$ & 0.83 & 1568.9 & 893.8 & 0.0 \\
\hline Average & 83 & 5 & 281 & 2106.3 & $<1$ & 17.1 & 1835.4 & $<1$ & 2.66 & 1829.5 & $<1$ & 2.15 & 1807.2 & 221.4 & 0 \\
\hline
\end{tabular}

The average RPD over 300 problem instances indicates that the ACS and EST has the best performance over the existing SS heuristic and HH heuristic. The average RPD of ACS and ETS heuristic is noted as 0\% and $2.15 \%$ whereas the RPD value of SS heuristic and $\mathrm{HH}$ heuristic are noted as $2.66 \%$ and $17.1 \%$ respectively.

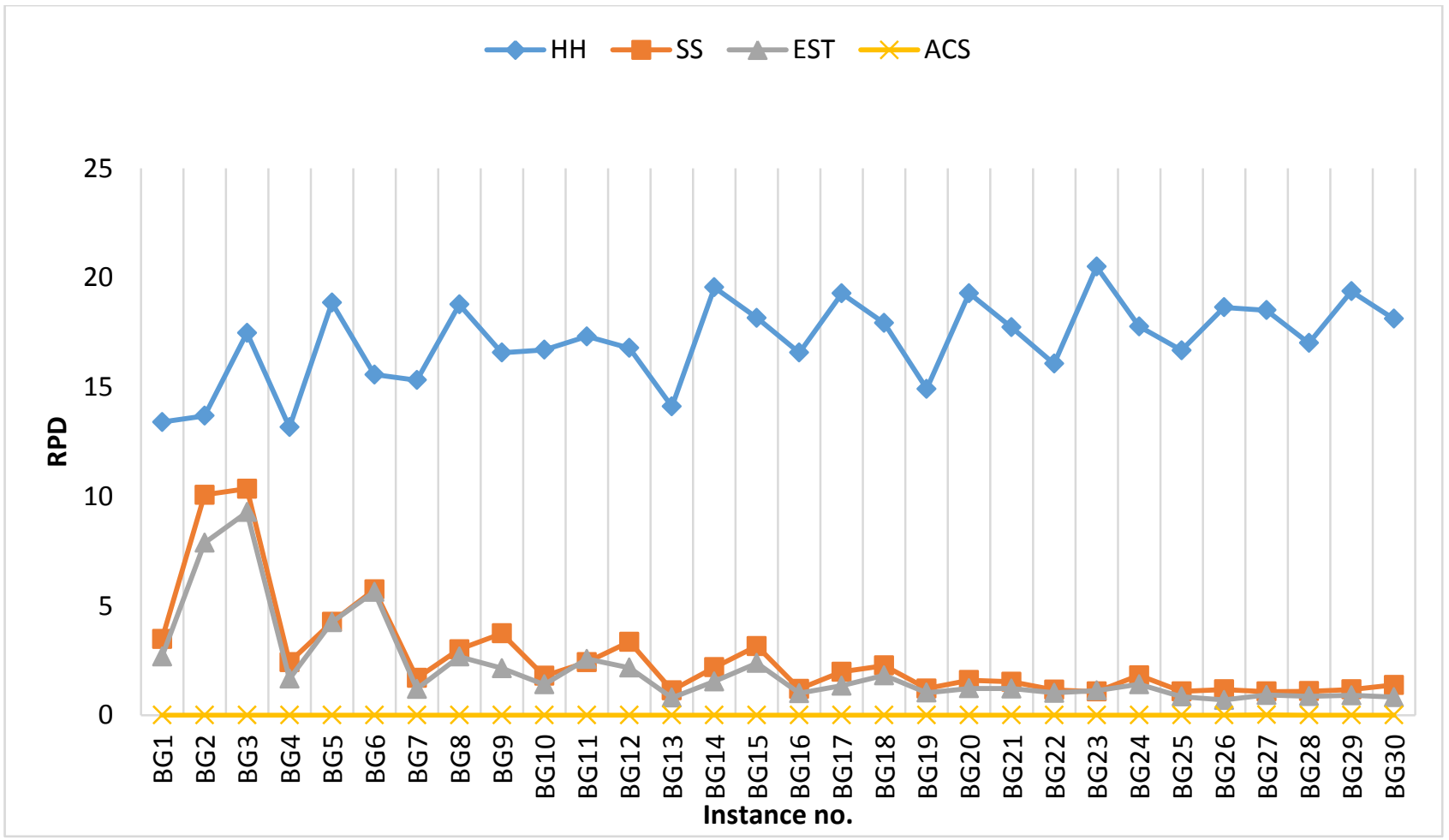

Figure 9: RPD of Algorithms with Makespan objective function for large data set. 
The performance of ACS is better compared to the performance of EST, SS and HH heuristics in all 120 problem instances. Another interesting observation about the CPU time of ACS can be made from Tables 5 and 6. The CPU time of ACS for Makespan objective is lower than the TCT objective. The similar trend is also observed for the CPU time of optimal solution for solving Makespan and TCT objective function. These results indicate that solving Makespan objective is easier than solving TCT objective.

Figure 10 shows the comparison of scheduling time for algorithms with large dataset and ACS performs better than EST, SS and HH. ACS has 16\%, 18\% and 19\% less than EST, SS and HH respectively. The reason behind better performance of ACS is the implementation of precedence task constraints during task scheduling.

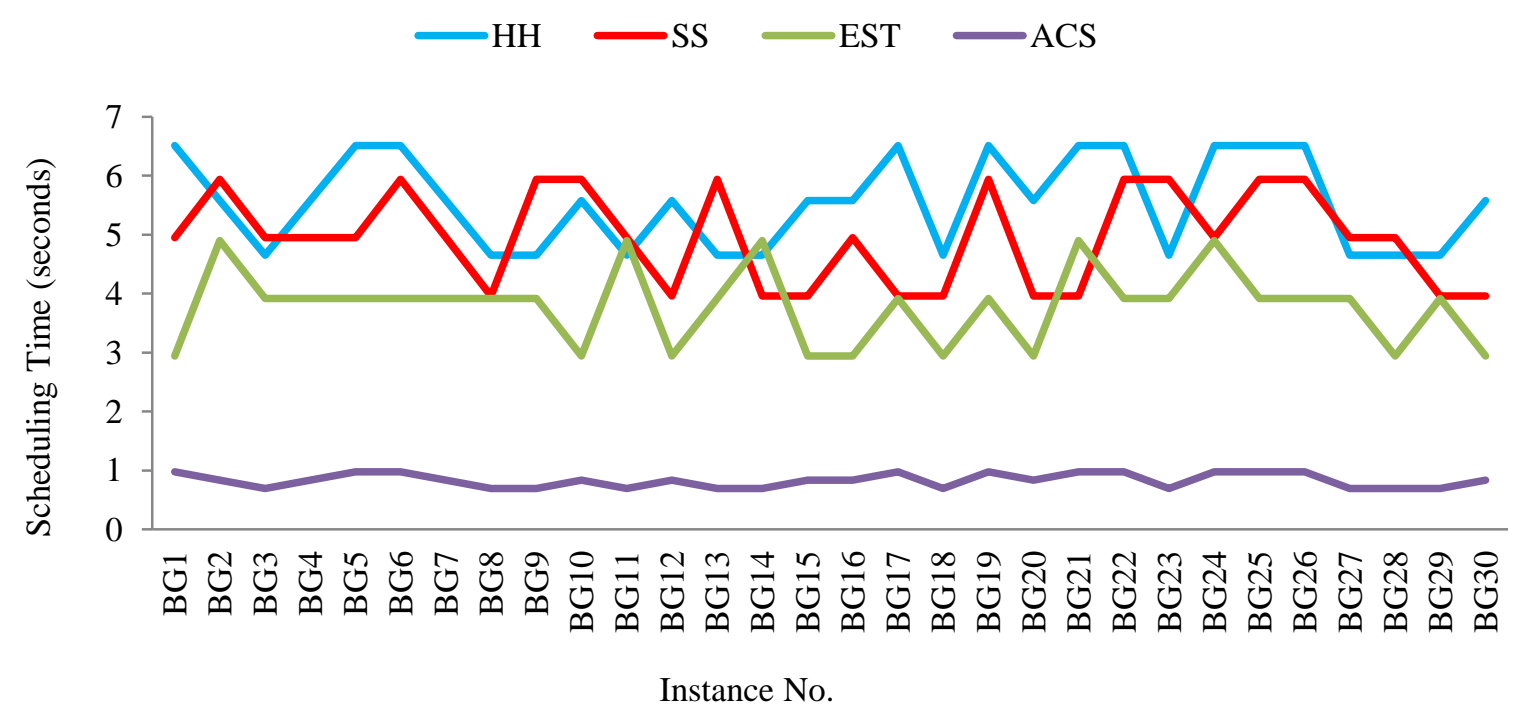

Figure 10: Comparison of scheduling time for algorithms with large dataset

\section{Conclusions and Future Scope}

A cloud computing scheduling problem is considered in this paper where jobs are processed in the parallel computing resources with precedence constraints. The tasks consist of many interdependent subtasks that can be processed in one of the unrelated parallel computing resources. This kind of problem is considered as an NP hard problem. For NP- hard problem, the heuristics and metaheuristics solutions become an obvious 
choice as heuristics and metaheuristics promise a suitable solution method. In this paper, two existing heuristic $\mathrm{HH}$ and SS are considered as most relevant heuristics for this specific problem domain. The existing heuristic did not utilize the scheduling algorithm property of unrelated parallel machine scheduling problem. The SS heuristic use earliest available machine assignment rule. This paper shows that the earliest available machine assignment rule is non dominant for the unrelated parallel machine problem with precedence constraints. This paper proposes an EST heuristic and an ACS metaheuristic which utilize the list scheduling algorithm property of the problem. The experimental results reveal the superior performance of proposed EST heuristic over existing heuristics. The use of list scheduling algorithm helps proposed heuristic to perform better than existing heuristic. The experimental results also indicate that solving Makespan objective is easier than solving TCT objective. The performance of ACS is found to be best for minimizing the Makespan objective as well as minimizing the total completion time objective and scheduling time. ACS shows the promising results which will help the cloud computing service providers to render the quality services for its end user cloud service users.

Future directions include implementation and testing in a distributed Fog-Cloud setup using FogBus framework provided by Tuli et al. (2019). To achieve this, data sharing techniques need to be tested to ensure that the ACS algorithm works seamlessly in a distributed setup. We also propose to extend this work to more sophisticated environments wherein we consider the myriad of factors crucial in a large-scale cloud/grid setup. Such factors include geographic distance, costs of the machine, network bandwidth for communication, resource utilization, etc. We will explore scalability and model limitations of proposed work in the future. Further research directions include integration of ensemble methods (Tuli et al. 2020) to achieve low scheduling times and explore other optimization methods like the ones using Pareto efficiency to test the robustness of the proposed algorithms. 


\section{Acknowledgements}

We thank Prof. Satish N. Srirama (Editor) and anonymous reviewers for their valuable comments and suggestions for improving our research paper. This research is partially supported by NSERC discovery Grant 318689.

\section{References}

Afzalirad M. \& Rezaeian J. (2016). Resource-constrained unrelated parallel machine scheduling problem with sequence dependent setup times, precedence constraints and machine eligibility restrictions, Computers \& Industrial Engineering 98 (2016) 40-52.

Buyya, Rajkumar, Satish Narayana Srirama, Giuliano Casale, Rodrigo Calheiros, Yogesh Simmhan, Blesson Varghese, Erol Gelenbe et al. "A manifesto for future generation cloud computing: Research directions for the next decade." ACM computing surveys (CSUR) 51, no. 5 (2018): 1-38.

Arnaout, J.-P., Rabadi, G., \& Musa, R. (2010). A two-stage ant colony optimization algorithm to minimize the makespan on unrelated parallel machines with sequence-dependent setup times. Journal of Intelligent Manufacturing, 21(6), 693-701.

Tuli, Shikhar, and Shreshth Tuli. "AVAC: A Machine Learning based Adaptive RRAM Variability-Aware Controller for Edge Devices." arXiv preprint arXiv:2005.03077 (2020).

Behnamian, J., Zandieh, M., \& Ghomi, S. F. (2009). Parallel-machine scheduling problems with sequencedependent setup times using an ACO, SA and VNS hybrid algorithm. Expert Systems with Applications, 36(6), 9637-9644.

Cheng C.Y. \& Huang L.W. (2017) Minimizing total Earliness and Tardiness through Unrelated parallel Machine scheduling Using distributed Release time Control, Journal of Manufacturing Systems 42 (2017) 110

Gill, Sukhpal Singh, Shreshth Tuli, Minxian Xu, Inderpreet Singh, Karan Vijay Singh, Dominic Lindsay, Shikhar Tuli et al. "Transformative effects of IoT, Blockchain and Artificial Intelligence on cloud computing: Evolution, vision, trends and open challenges." Internet of Things 8 (2019): 100118.

Cook, S. A. (1971). The complexity of theorem-proving procedures. Paper presented at the Proceedings of the third annual ACM symposium on Theory of computing. 
Dutta, D., \& Joshi, R. (2011). A genetic: algorithm approach to cost-based multi-QoS job scheduling in cloud computing environment. Paper presented at the Proceedings of the International Conference \& Workshop on Emerging Trends in Technology.

Fang, Y., Wang, F., \& Ge, J. (2010). A task scheduling algorithm based on load balancing in cloud computing. Paper presented at the International Conference on Web Information Systems and Mining.

Gajpal, Y., Rajendran, C. (2006). An ant-colony optimization algorithm for minimizing the completion-time variance of jobs in flowshops, International Journal of Production Economics, 101(2), 259-272.

Tuli, Shreshth, Shashikant Ilager, Kotagiri Ramamohanarao, and Rajkumar Buyya. "Dynamic Scheduling for Stochastic Edge-Cloud Computing Environments using A3C learning and Residual Recurrent Neural Networks." Transaction on Mobile Computing 2020. https://doi.org/10.1109/TMC.2020.3017079

Gao, Y., Guan, H., Qi, Z., Hou, Y., \& Liu, L. (2013). A multi-objective ant colony system algorithm for virtual machine placement in cloud computing. Journal of Computer and System Sciences, 79(8), 1230-1242.

Gacias B, Artigues C \& Lopez P. (2010). Parallel machine scheduling with precedence constraints and setup times, Computers \& Operations Research, 37(12), pp. 2141-2151.

Garey, M. R., \& Johnson, D. S. (1979). Computers and intractability: a guide to the theory of NPcompleteness. 1979. San Francisco, LA: Freeman, 58.

Germain-Renaud, C., Rana, O. (2009).The convergence of clouds, grids, and autonomics, IEEE Internet Computing 13 (6).

Herrmann, J., Proth, M., \& Sauer, N. (1997). Heuristics for unrelated machine scheduling with precedence constraints, European Journal of Operational Research, (102), 528-537.

Hong, J., Diabat, A., Panicker, V., V., Rajagopalan, S.,(2018). A two-stage supply chain problem with fixed costs: An ant colony optimization approach, , International Journal of Production Economics, Vol. 204, 214226.

Hua, X.-y., Zheng, J., \& Hu, W.-x. (2010). Ant colony optimization algorithm for computing resource allocation based on cloud computing environment [J]. Journal of East China Normal University (Natural Science), 1(1), 127-134.

Hurink, J., Knust, S. (2001). List scheduling in a parallel machine enviornment with precedence constraints and setup times, Operation Research Letters, (29), 231-239.

Islam, Muhammed Tawfiqul, Satish Narayana Srirama, Shanika Karunasekera, and Rajkumar Buyya. "Costefficient dynamic scheduling of big data applications in apache spark on cloud." Journal of Systems and Software 162 (2020): 110515. 
Jang, S. H., Kim, T. Y., Kim, J. K., \& Lee, J. S. (2012). The study of genetic algorithm-based task scheduling for cloud computing. International Journal of Control and Automation, 5(4), 157-162.

Joo C.M., Kim B.S., (2015). Hybrid genetic algorithms with dispatching rules for unrelated parallel machine scheduling with setup time and production availability, Computers \& Industrial Engineering, (85),102-109.

Jose Elias C. , Joseph Y., T. Leung. (2017) Scheduling unrelated parallel batch processing machines, Computers \& Operations Research, (78), 117-128.

Kaur, Amanpreet, V. P. Singh, and Sukhpal Singh Gill. "The future of cloud computing: opportunities, challenges and research trends." In 2018 2nd International Conference on I-SMAC (IoT in Social, Mobile, Analytics and Cloud) pp. 213-219. IEEE, 2019.

Kochan, C., G., Nowicki, D., R., Sauser, B., Randall, W., S., (2018), Impact of cloud-based information sharing on hospital supply chain performance: A system dynamics framework, International Journal of Production Economics, Vol. 195, 168-185.

Li, J.-F., \& Peng, J. (2011). Task scheduling algorithm based on improved genetic algorithm in cloud computing environment. Jisuanji Yingyong/ Journal of Computer Applications, 31(1), 184-186.

Li j., Qiu, M., Mingb, Z., Quan, G., Qin, X., and Gu, Z. (2012). Online optimization for scheduling preemptable tasks on IaaS cloud systems, J. Parallel Distrib. Comput. 72 (2012) 666-677.

Lin, Y. K., Pfund, M. E., \& Fowler, J. W. (2011). Heuristics for minimizing regular performance measures in unrelated parallel machine scheduling problems. Computers \& Operations Research, 38(6), 901-916.

Lin, Y. K., Pfund, M. E., \& Fowler, J. W. (2013). Multiple-objective heuristics for scheduling unrelated parallel machines, European Journal of Operational Research 227 (2013) 239-253.

Liu, J., Luo, X.-G., Zhang, X.-M., Zhang, F., \& Li, B.-N. (2013). Job scheduling model for cloud computing based on multi-objective genetic algorithm. International Journal of Computer Science Issues, 10(1), 134139.

Liu, C., \& Yang, S. (2011). A heuristic serial schedule algorithm for unrelated parallel machine scheduling with precedence constraints, Journal of Software, Vol. 6, No. 6, pp 1146-1153.

Luis, F-P., Ruiz, R. (2010). Iterated greedy local search methods for unrelated parallel machine scheduling. European Journal of Operational Research 207, (2010) 55-69.

Luis F-P., Perea, F., \& Ruiz, R. (2017). Models and matheuristics for the unrelated parallel machine scheduling problem with additional resources, European Journal of Operational Research, 260 (2017) 4 82-4 93. 
Oleh Sobeyko, Lars Mönch Heuristic approaches for scheduling jobs in large-scale flexible job shops, Computers \& Operations Research 68 (2016) 97-109

Pandey, S., Wu, L., Guru, S. M., \& Buyya, R. (2010). A particle swarm optimization-based heuristic for scheduling workflow applications in cloud computing environments. Paper presented in the Advanced information networking and applications (AINA), 24th IEEE international conference.

Tuli, Shreshth, Redowan Mahmud, Shikhar Tuli, and Rajkumar Buyya. "Fogbus: A blockchain-based lightweight framework for edge and fog computing." Journal of Systems and Software 154 (2019): 22-36.

Rodney Oliveira Marinho Diana, Moacir Felizardo de França Filho, Sérgio Ricardo de Souza, João Francisco de Almeida Vitor. (2015) An immune-inspired algorithm for an unrelated parallel machines' scheduling problem with sequence and machine dependent setup-times for makespan minimisation, Neurocomputing, Volume 163, 2015, pp. 94-105.

Saif U. R. Malik, Hina Akram, Sukhpal Singh Gill, Haris Pervaiz, Hassan Malik. EFFORT: Energy efficient framework for offload communication in mobile cloud computing. Software: Practice and Experience Wiley, 2020;1-14. https://doi.org/10.1002/spe.2850

Shahvari O., Logendran R., An Enhanced tabu search algorithm to minimize a bi-criteria objective in batching and scheduling problems on unrelated-parallel machines with desired lower bounds on batch sizes, Computers \& Operations Research, 77, (2017), 154-176.

Sukhpal Singh Gill \& Rajkumar Buyya. Resource Provisioning Based Scheduling Framework for Execution of Heterogeneous and Clustered Workloads in Clouds: from Fundamental to Autonomic Offering, J Grid Computing (2019). Volume 17, Number 3, Pages: 385-417, 2019

Thomas Stützle, T., Hoos, H., H. (2000). Max-Min ant System, Future generation computer systems, 16(8), 889-914.

Thepphakorn, T., Pongcharoen, P., Hicks, C., (2014). An ant colony based timetabling tool, International Journal of Production Economics, Vol. 149, 131-144.

Tuli, Shreshth, Nipam Basumatary, Sukhpal Singh Gill, Mohsen Kahani, Rajesh Chand Arya, Gurpreet Singh Wander, and Rajkumar Buyya. "Healthfog: An ensemble deep learning based smart healthcare system for automatic diagnosis of heart diseases in integrated iot and fog computing environments." Future Generation Computer Systems 104 (2020): 187-200.

Thiruvady, D., Singh, G., Ernst, A., T., Meyer, B., (2013). Constraint-based ACO for a shared resource constrained scheduling problem, International Journal of Production Economics, Vol. 141, 230-242. 
Ting, C., J., and Chen, C., H., (2013). A multiple ant colony optimization algorithm for the capacitated location routing problem, International Journal of Production Economics, Vol. 141, 34-44.

Tsai, J.T., Fang, J., \& Chou, J.H. (2013). Optimized task scheduling and resource allocation on cloud computing environment using improved differential evolution algorithm. Computers \& operations research, 40, 3045-3055.

Zhang, S., Gajpal, Y., Appadoo, Abdulkader, M.M.S. (2018). Electric vehicle routing problem with recharging stations for minimizing energy consumption, International Journal of Production Economics, Vol. 203, 404-413.

Enterprise Cloud Computing Survey, https://clutch.co/cloud\#survey, accessed 9 June 2017.

The Changing of Faces the Cloud, http://www.bain.com/Images/BAIN_BRIEF_The_Changing_Faces_of_the_Cloud.pdf, accessed 9 June 2017. Wang, Wang, and Yu Cheng. "Optimal Charging Scheduling for Electric Vehicles Considering the Impact of Renewable Energy Sources." In 2020 5th Asia Conference on Power and Electrical Engineering (ACPEE), pp. 1150-1154. IEEE, 2020.

Deng, Runze, Fengji Luo, Gianluca Ranzi, Zehua Zhao, and Yan Xu. "A MILP Based Two-Stage Load Scheduling Approach for Building Load's Peak-to-Average Ratio Reduction." In 2020 5th Asia Conference on Power and Electrical Engineering (ACPEE), pp. 771-775. IEEE, 2020.

Gill, Sukhpal Singh, Shreshth Tuli, Adel Nadjaran Toosi, Felix Cuadrado, Peter Garraghan, Rami Bahsoon, Hanan Lutfiyya et al. "ThermoSim: Deep learning based framework for modeling and simulation of thermalaware resource management for cloud computing environments." Journal of Systems and Software. 166 (2020): 110596.

Srirama, Satish Narayana, and Alireza Ostovar. "Optimal cloud resource provisioning for auto-scaling enterprise applications." International Journal of Cloud Computing 7, no. 2 (2018): 129-162.

Zhou, Bowen, Amir Vahid Dastjerdi, Rodrigo N. Calheiros, Satish Narayana Srirama, and Rajkumar Buyya. "mCloud: A context-aware offloading framework for heterogeneous mobile cloud." IEEE Transactions on Services Computing 10, no. 5 (2015): 797-810.

Casini, Daniel, Alessandro Biondi, and Giorgio Buttazzo. "Timing isolation and improved scheduling of deep neural networks for real-time systems." Software: Practice and Experience. (2020). https://doi.org/10.1002/spe.2840

Khiat, Abdelhamid, Abdelkamel Tari, and Tom Guérout. "MFHS: A modular scheduling framework for heterogeneous system." Software: Practice and Experience (2020). https://doi.org/10.1002/spe.2827 
Wainer, Gabriel, and Mohammad Moallemi. "Designing real-time systems using imprecise discrete-event system specifications." Software: Practice and Experience (2020). https://doi.org/10.1002/spe.2831

Bhatt, Ashutosh, Priti Dimri, and Ambika Aggarwal. "Self-adaptive brainstorming for jobshop scheduling in multicloud environment." Software: Practice and Experience (2020). https://doi.org/10.1002/spe.2819

Ezugwu, Absalom E. "Enhanced symbiotic organisms search algorithm for unrelated parallel machines manufacturing scheduling with setup times." Knowledge-Based Systems 172 (2019): 15-32.

Vallada, Eva, Fulgencia Villa, and Luis Fanjul-Peyro. "Enriched metaheuristics for the resource constrained unrelated parallel machine scheduling problem." Computers \& Operations Research 111 (2019): 415-424.

Lei, Deming, and Meiyao Liu. "An artificial bee colony with division for distributed unrelated parallel machine scheduling with preventive maintenance." Computers \& Industrial Engineering 141 (2020): 106320.

Hunkeler, Urs, Hong Linh Truong, and Andy Stanford-Clark. "MQTT-S—A publish/subscribe protocol for Wireless Sensor Networks." In 2008 3rd International Conference on Communication Systems Software and Middleware and Workshops (COMSWARE'08), pp. 791-798. IEEE, 2008. 